\title{
Stable heteroclinic cycles and symbolic dynamics
}

\author{
Lluís Alsedà \\ Departament de Matemàtiques, Universitat Autònoma de Barcelona, 08193-Bellaterra, Barcelona, Spain \\ Jean Marc Gambaudo \\ I.N.L.N. URM CNRS 129, Université de Nice-Sophia Antipolis, 1361, Route des Lucioles, Sophia Antipolis, \\ 06560 Valbonne, France \\ Pere Mumbrú \\ Departament de Matemàtica Aplicada i Anàlisi, Universitat de Barcelona, Gran Via 585, \\ 08071-Barcelona, Spain
}

(Received 23 March 1993; accepted for publication 10 January 1994)

Let $\mathbf{S}_{0}^{1}, \mathbf{S}_{1}^{1}, \ldots, \mathbf{S}_{n-1}^{1}$ be $n$ circles. A rotation in $n$ circles is a map $f: \cup_{i=0}^{n-1} \mathbf{S}_{i}^{1} \rightarrow \cup_{i=0}^{n-1} \mathbf{S}_{i}^{1}$ which maps each circle onto another by a rotation. This particular type of interval exchange map arises naturally in bifurcation theory. In this paper we give a full description of the symbolic dynamics associated to such maps.

\section{INTRODUCTION}

In bifurcation theory, we often have to deal with a critical vector field in $\mathbf{R}^{m}, m \geqslant 2$, which satisfies the following properties: there exists a finite sequence of singularities, $p_{0}$, $p_{1}, \ldots, p_{n-1}$, of saddle type with only one unstable direction, and a sequence of $2 n$ heteroclinic or homoclinic orbits, $\Gamma_{0,0}$, $\Gamma_{0,1}, \ldots, \Gamma_{i, 0}, \Gamma_{i, 1}, \ldots, \Gamma_{n-1,0}, \Gamma_{n-1,1}$, joining one of the $p_{i}$ 's to another. Such a configuration is called a heteroclinic cycle (see Fig. 1). It turns out that, in many situations, the set $\Gamma=$ $\overline{\cup_{i=0}^{n-1}\left(\Gamma_{i, 0} \cup \Gamma_{i, 1}\right)}$ is an attractor. In such a case, we call it a stable heteroclinic cycle.

Stable heteroclinic cycles are obviously not structurally stable, and consequently the following question arises:

Question: Consider a $\%^{1}$ vector field in $\mathbf{R}^{m}, m \geqslant 2$, with a stable heteroclinic cycle $\Gamma$. What happens in a neighborhood of $\Gamma$ when we perturb the vector field in the $\mathscr{V}^{1}$ topology?

This question has been completely answered, in the particular case called "gluing bifurcation," of a configuration involving only one singularity. ${ }^{1-3}$

However, so far, there is no answer in the general case, which remains very interesting for the following two reasons:

First, it is not an academic generalization of the gluing bifurcation: there exist some extra difficulties related to the increasing richness of the possible dynamical behavior:

Second, stable heteroclinic cycles (with more than one singularity) occur in problems of bifurcation coming from the PDEs world (for instance hydrodynamics ${ }^{4}$ ).

In this paper we focus our attention to a particular class of (stable) heteroclinic cycle. These cycles will be called rotating (stable) heteroclinic cycles (see Fig. 2) and correspond to the case when the two heteroclinic or homoclinic orbits $\Gamma_{i, 0}$ and $\Gamma_{i, 1}$, emanating from a singularity $p_{i}$, end at the same singularity $p_{j}$.

Consider now a $\mathscr{C}^{1}$ vector field $X_{0}$ in $\mathbf{R}^{m}$ with a rotating stable heteroclinic cycle $\Gamma$ and assume that the linearized vector field $D X_{0}\left(p_{i}\right)$, at each singularity $p_{i}$, is such that its dominating stable eigenvalue is real. Then, generically, there exists a rigorous way to reduce the dynamics of any vector field $X, \mathscr{Y}^{1}$-close to $X_{0}$ in a neighborhood of $\Gamma$, to the dynamics of a map $f_{[X]}$ on the interval with a finite number of discontinuities (see Fig. 3). Furthermore, this map is monotone and is a contraction of each interval of continuity. This reduction to a one-dimensional dynamics is the subject of Sec. II.

Some simple extra conditions on the vector field $X_{0}$ yields maps $f_{[X]}$ from the union of $n$ intervals into itself, mapping one interval into another with a single discontinuity. in each interval.

For a vector field $X, \gamma^{1}$-close to $X_{0}$, there is a natural way of coding the invariant curves of $X$ which remain in a neighborhood of $\Gamma$.

Roughly speaking, we can code an invariant curve with a periodic sequence of $2 n$ symbols corresponding to the fact that the curve has to follow successively some of the $2 n$ heteroclinic or homoclinic orbits of $X_{0}$. In Sec. III, we show how this coding corresponds, on the interval, to the classical Milnor-Thurston coding for the periodic orbits of the map $f_{[X]}$.

We show also that each code of a periodic orbit of $f_{[X]}$ is also the code of the periodic orbit of a map $f_{[X]}^{*}$ which also has one discontinuity in each interval, the same monotonicity type of $f_{[X]}$ but which is an interval exchange.

When this interval exchange preserves the orientation it can be seen as a map from the union of $n$ circles into itself, which maps each circle onto another by a rotation. We call this type of map a composition of rotations in $n$ circles (see Fig. 4 for an example of these maps). Sections IV-VIII are devoted to the study of the symbolic dynamics of these maps:

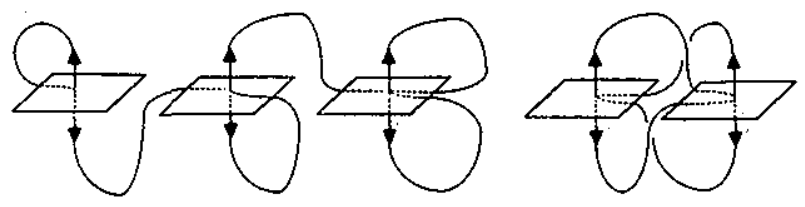

FIG. 1. Some heteroclinic cycles. 


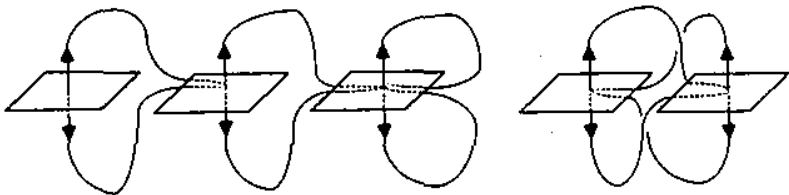

FIG. 2. Somc rotating heteroclinic cycles.

\section{ROTATING STABLE HETEROCLINIC CYCLES}

We note that most of the techniques used in this section are now quite standard. We refer to Refs. 1 and 2 for the details of quite similar proofs.

Let $X_{0}$ be a $\zeta^{1}$ vector field in $\mathbf{R}^{m}, m \geqslant 2$, such that:

Hypothesis $\mathbf{H}_{1}$. $X_{0}$ possesses $n$ hyperbolic saddle singularities $p_{0}, p_{1}, \ldots, p_{n-1}$;

Hypothesis $\mathbf{H}_{2}$. The linearized vector field $D X_{0}\left(p_{i}\right)$ at each singularity $p_{i}$, after a smooth change of coordinates, reads

$$
\begin{aligned}
D X_{0}\left(p_{i}\right)= & \lambda_{i, 1} x_{1} \frac{\partial}{\partial x_{1}}+\lambda_{i, 2} x_{2} \frac{\partial}{\partial x_{2}} \\
& +\sum_{j=2}^{j=m} \sum_{k=2}^{k=m} \mu_{j k} x_{k} \frac{\partial}{\partial x_{j}},
\end{aligned}
$$

where

$$
0<\lambda_{i, 1}<-\lambda_{i, 2},
$$

and

$$
\lambda_{i, 2}=\max \operatorname{Re} \lambda_{i, k} ; \quad \lambda_{i, k} \in \operatorname{Spec} D X_{0}\left(p_{i}\right)-\left\{\lambda_{i, 1}\right\} .
$$

Hypothesis $\mathbf{H}_{3}$. There exists a map $\tau:\{0,1, \ldots, n-1\}$ $\rightarrow\{0,1, \ldots, n-1\}$ such that, for each $i \in\{0,1, \ldots, n-1\}$, the two homoclinic or heteroclinic orbits $\Gamma_{i, 1}$ and $\Gamma_{i, 0}$ emanating from $p_{i}$ end at $p_{\tau(i)}$. To fix the notations, we can assume that $\Gamma_{i, 1}$ (resp. $\Gamma_{i, 0}$ ) is the orbit starting at $p_{i}$ on the side $x_{1}>0$ (resp. $x_{1}<0$ ).

Hypothesis $\mathbf{H}_{4}$. We know that, with the above conditions, both orbits $\Gamma_{i, 0}$ and $\Gamma_{i, 1}$ arrive to $p_{\tau(i)}$ tangent to the line $x_{1}=x_{3}=\cdots=x_{m}=0$. In addition to this generic condition we assume that these two orbits arrive to $p_{\tau(i)}$ at the same side: $x_{2}>0$.

It is very easy to check that, thanks to the conditions we have given on the eigenvalues of the linearized vector field at

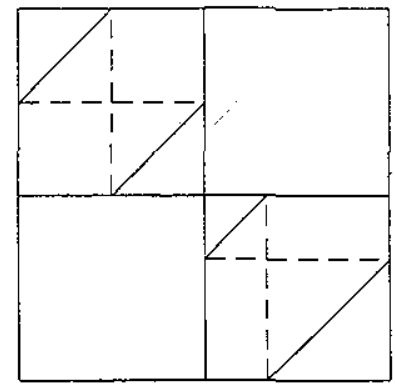

FIG. 4. A composition of rotations in two circles.

each singularity, the rotating heteroclinic cycle $\Gamma$ we have defined is stable (see Refs. 1 and 2). More precisely, let $\varphi_{0}(\cdot, t)$ be the "time $t$ " map of the flow induced by $X_{0}$. Then, there exists a neighborhood $U$ of $\Gamma$ such that

$$
\varphi_{0}(U, t) \subset U \text { for } t \geqslant 0
$$

and

$$
\Gamma=\bigcap_{t \geqslant 0} \varphi_{0}(U, t)
$$

Furthermore, this neighborhood is also invariant by the "time $t$ " $(t \geqslant 0)$ map of the flow of any vector field $\mathscr{Y}^{1}$-close to $X_{0}$.

Thanks to the hyperbolicity of the singularities $p_{i}$, and up to a smooth change of coordinates, it is not restrictive to assume that for any vector field $\varnothing^{1}$-close to $X_{0}$, the points $p_{0}, p_{1}, \ldots, p_{n-1}$ remain hyperbolic singularities, the local stable manifold is given by the equation $x_{1}=0$ and the local unstable manifold by the equations $x_{2}=x_{3}=\cdots=x_{m}=0$.

For $h$ and $r$ positive and small enough consider, near each singularity $p_{i}$, the rectangle $R_{i}$ defined by $x_{2}=h$ and $\left|x_{j}\right|<r$ for $j=1,3, \ldots, m$ (see Fig. 5).

By following the flow induced by $X_{0}$, the rectangles $R_{i, 0}=R_{i} \cap\left\{x_{1}<0\right\}$ and $R_{i, 1}=R_{i} \cap\left\{x_{1}>0\right\}$ are mapped in

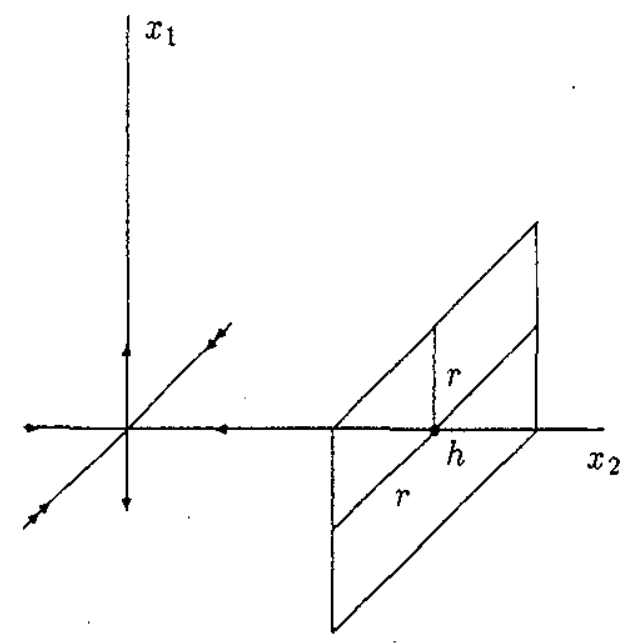




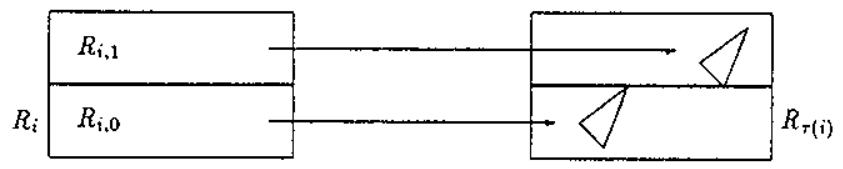

FIG. 6. The standard situation for $X_{0}$.

$R_{\tau(i)}$ on two singular triangles whose singular extremities lie on $x_{1}=0$ (see Fig. 6). These points correspond to the intersection of $\Gamma_{i, 0}$ and $\Gamma_{i, 1}$ with $R_{\tau(i)}$.

For a perturbed vector field $X, \mathscr{C}^{1}$-close to $X_{0}$, we have the same situation except that the two branches of the unstable manifold at $p_{i}$ do not need to cross $R_{\tau(i)}$ on the line $x_{i}=0$ (see Fig. 7). In other words, we may not have a heteroclinic or a homoclinic connection.

The two maps we have constructed from $R_{i, 0}$ to $R_{\tau(i)}$ and from $R_{i, 1}$ to $R_{\tau(i)}$ can be extended by continuity to maps from $\bar{R}_{i, 0}$ to $R_{\tau(i)}$ and from $\bar{R}_{i, 1}$ to $R_{\tau(i)}$, where $\tilde{R}_{i, 0}=R_{i} \cap\left\{x_{1} \leqslant 0\right\}$ and $\bar{R}_{i, 1}=R_{i} \cap\left\{x_{1} \geqslant 0\right\}$. This yields a map $T_{[X], i}: R_{i} \rightarrow R_{\tau(i)}$ which is bivalued on the line $x_{1}=0$.

From Hypothesis $\mathrm{H}_{2}$, it is easy to check that the maps $T_{[X], i}$ restricted to $\bar{R}_{i, 0}$ and $\bar{R}_{i, 1}$ are contractions.

We set

$$
T_{[X]}: \bigcup_{i=0}^{n-1} R_{i} \rightarrow \bigcup_{i=0}^{n-1} R_{i}
$$

by $\left.T_{[X]}\right|_{R_{i}}=T_{[X], i}$.

Let us now assume the following generic condition.

Hypothesis $\mathbf{H}_{5}$. The images by $T_{[X], i}$ of the two arcs

$$
\left.\gamma_{i, 1}(t)=\left\{\left(x_{1}, \ldots, x_{m}\right)\right\} \mid x_{1}=t \geqslant 0, x_{2}=h, x_{i}=0, i \geqslant 3\right\}
$$

and

$$
\left.\gamma_{i, 0}(t)=\left\{\left(x_{1}, \ldots, x_{m}\right)\right\} \mid x_{1}=t \leqslant 0, x_{2}=h, x_{i}=0, i \geqslant 3\right\}
$$

are not tangent at $t=0$ to the hyperplane $x_{1}=0$.

With this supplementary condition, we can prove (see Ref. 1) that in each $R_{i}$ there exists a $\mathscr{C}^{d}$-codimension 1 foliation invariant by $T_{[X]}$ (this foliation contains the leaves $\left.x_{1}=0\right)$.

The dynamics on the leaves is a contraction and, on the quotient space (which is one dimensional) we are reduced to a map $f_{[X]}$ on the union of $n$ intervals $I_{i}=[-r, r]$ such that $f_{[X]}\left(I_{i}\right) \subset I_{\tau(i)}$ and

$$
\begin{aligned}
f_{[X], i}(x) & \equiv f_{[X]} \mid I_{i}(x) \\
& = \begin{cases}\mu_{i, 1}+c_{i, 1} x^{\alpha_{i}}+\text { h.o.t. } & \text { if } x \geqslant 0, \\
\mu_{i, 0}+c_{i, 0} x^{\alpha_{i}}+\text { h.o.t. } & \text { if } x \leqslant 0,\end{cases}
\end{aligned}
$$

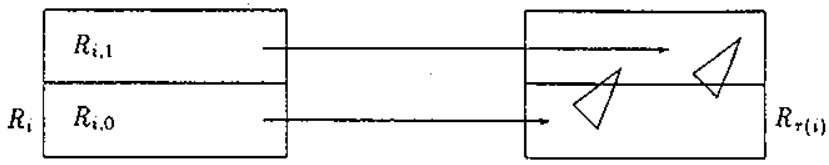

FIG. 7. The standard situation for $X, \mathscr{Y}^{1}$ close to $X_{0}$.

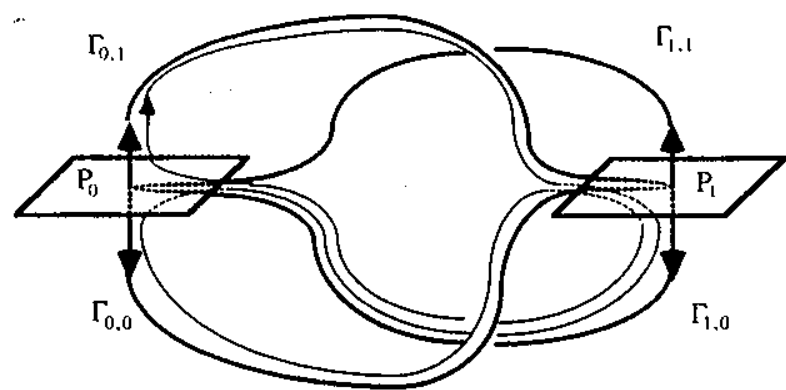

FIG. 8. A simple oriented closed curve with code $(0,1)(1,0)(0,0)(1,0)$.

where $\alpha_{i}=-\lambda_{i, 2} / \lambda_{i, 1}$ (recall that the neighborhood $U$ and consequently $r$ have to be chosen small enough so that $\left.f_{[X], i}\right|_{\{r \leqslant 0\}}$ and $\left.f_{[X], i}\right|_{\{r \geqslant 0\}}$ are contractions).

\section{SYMBOLIC DYNAMICS}

Consider a vector field $X_{0}$ with a stable heteroclinic cycle $\Gamma$, and let $U$ be a small tubular neighborhood of $\Gamma$. Any oriented simple closed curve in $U$ yields, by retraction, an oriented loop in $\Gamma$. This loop is homotopic to a loop consisting on a succession of arcs $\Gamma_{i, 0}$ or $\Gamma_{i, 1}$ followed in a positive (time increasing) or negative direction. Consequently, to any oriented simple closed curve in $U$ we can associate a periodic sequence $\left(\ldots x_{0} x_{1} \ldots x_{l} x_{0} \ldots\right)$ of symbols in $\{-1,1\} \times\{0,1, \ldots, n$ $-1\} \times\{0,1\}$. If this simple closed curve is an invariant curve of a vector field $\mathscr{C}^{1}$-close to $X_{0}$, then the coding is simpler because there is a natural orientation of the orbits in $U$. It follows that the corresponding sequence will be in $\{1\}$ $\times\{0,1, \ldots, n-1\} \times\{0,1\}$. Consequently, we can forget about the symbol $\{1\}$ which means the time increasing direction and associate, to each invariant curve $Y$ in $U$ of a vector field $\mathscr{C}^{2}$-close to $X_{0}$, a sequence of symbols $I(Y)=\left\{y_{l}\right\}$ where the $y_{l}$ 's belong to $\{0,1, \ldots, n-1\} \times\{0,1\}$ (see Fig. 8 for an example).

Assume now that the vector field $X$ satisfies the assumptions from $\mathrm{H}_{1}$ to $\mathrm{H}_{5}$. A simple oriented closed curve in $U$ of a vector field $X, \mathscr{K}^{1}$-close to $X_{0}$, corresponds to a periodic orbit of $T_{[X]}$ and $f_{[X]}$.

This curve is a stable periodic orbit of $X$ if its corresponding periodic orbit of $T_{[X]}$ (resp. of $f_{[X]}$ ) avoids the line $x_{1}=0$ (resp. the point 0 in each $I_{i}$ ). If not, it is a homoclinic orbit (see Ref. 2).

To a periodic point $x$ of $T_{[X]}$ (resp. of $f_{[X]}$ ) we can associate a periodic sequence $\left(y_{l}\right)$ in the symbols $\{0,1, \ldots, n-1\}$ $\times\{0,1\}$ by setting $y_{l}=\left(m_{l}, \varepsilon_{l}\right)$ if and only if $T^{l}(x) \in R_{m_{l}, \varepsilon_{l}}$ (resp. $f^{l}(x) \in I_{l}$ and $f^{l}(x) \varepsilon_{l} \geqslant 0$ ).

It is straightforward to check that the code $I(Y)$ of an oriented closed invariant curve in $U$ of a vector field $X, \mathscr{E}^{1}$ close to $X_{0}$, coincides with the code of the corresponding periodic orbits of $T_{[X]}$ and $f_{[X]}$ defined above.

In the sequel, we are going to describe the different codes we can get for periodic orbits of the maps $f_{[X]}$. In order to do it, let us consider a periodic orbit $\theta$ of a map $f_{[X]}$.

By changing the position of the points of $\theta$ in the interval without changing their mutual order nor their positions with respect to zero, it is easy to see that there exists a map 


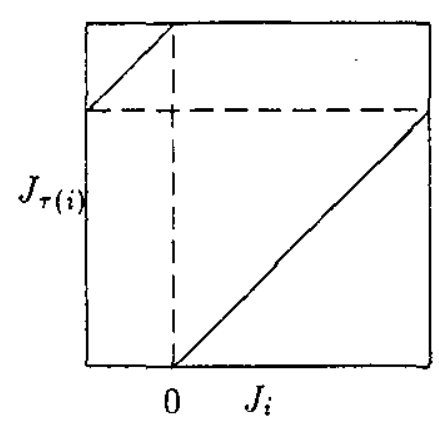

FIG. 9. The map $f_{[x]}^{*}$ when the hypotheses from $\mathrm{H}_{1}$ to $\mathrm{H}_{6}$ are fulfilled.

$$
f_{[X]}^{*}: \bigcup_{i=0}^{n-1} \vec{I}_{i} \rightarrow \bigcup_{i=0}^{n-1} \tilde{I}_{i}
$$

where $\tilde{I}_{i}$ are intervals which contain zero and satisfy the following conditions:

(i) $f_{[X]}^{*}$ possesses a discontinuity zero in each interval $\tilde{I}_{i}$;

(ii) maps $\tilde{I}_{i}$ onto $\tilde{I}_{\tau(i)}$;

(iii) it is an isometry on each interval of continuity (with the same monotonicity as $f_{[X]}$;

(iv) it possesses a periodic orbit with the same code (with respect to the new natural partition) as $\theta$.

By construction, this map $f_{[X]}^{*}$ is an interval exchange transformation.

To understand the symbolic dynamics of the invariant closed curves in $U$ of a vector field $\mathscr{C}^{1}$-close to $X_{0}$, it is thus necessary to understand the symbolic dynamics of some classes of interval exchanges transformations. This is a subject too vast and too rich. In the next section we are going to restrict our attention to the description of the symbolic dynamics associated to a special class of interval exchange transformations:

Hypothesis $\mathbf{H}_{6}$. Assume that on each interval of monotonicity the functions $f_{[X]}$ and $f_{[X]}^{*}$ are increasing and that $f_{[X], i}^{*}\left(0^{+}\right)<0<f_{[X], i}^{*}\left(0^{-}\right)$. Assume also that $\tau$ is a bijection and that the intervals $J_{i}$ $=\left[f_{[X], \tau^{-1}(i)}^{*}\left(0^{+}\right), f_{[X], \tau^{-1}(i)}^{*}\left(0^{-}\right)\right]$have all the same length and satisfy

$$
f_{[X]}^{*}\left(\bigcup_{i=0}^{n-1} J_{i}\right) \subset \bigcup_{i=0}^{n-1} J_{i}
$$

Notice that Hypothesis $\mathrm{H}_{6}$ is nothing more than conditions on the heteroclinic cycle $\Gamma$ ( $\tau$ bijective) and on the type of perturbed vector fields allowed. More precisely, one can think on the perturbations on the vector field in the following way. We can unfold the bifurcation diagram around the stable heteroclinic cycle in a $2 n$-parameter space. Each parameter corresponds to the breaking of a heteroclinic connection. In this setting, Hypothesis $\mathrm{H}_{6}$ restricts the allowed perturbations to a submanifold with dimension $n$ in the parameter space.

With all these restrictions, $f_{[X]}^{*}$ maps each interval $J_{i}$ onto $J_{\tau(i)}$ as follows (see Fig. 9):

(i) It possesses one discontinuity at 0 ; (ii) it is increasing with slope 1 on each interval of continuity;

(iii) it is surjective;

(iv) it is injective in the interior of $J_{i}$ but the two end points of $J_{i}$ have the same image.

By identifying the end points of the intervals $J_{i}$ we get a map $F_{[X]}$ from the union of $n$ circles $\mathbf{S}_{0}^{\mathrm{I}}, \mathbf{S}_{1}^{1}, \ldots, \mathbf{S}_{n-1}^{1}$ into itself (each of these circles has an origin 0 ) with the follow: ing properties. There exists a bijection $\tau:\{0,1, \ldots, n-1\}$ $\rightarrow\{0,1, \ldots, n-1\}$ and a map $\alpha:\{0,1, \ldots, n-1\} \rightarrow \mathbf{R}$ such that $F_{[X]}\left(\mathbf{S}_{i}^{1}\right)=\mathbf{S}_{\tau(i)}^{1}$ and $\wp_{\tau(i)}{ }^{\circ F_{[X]}} \mid \mathbf{S}_{i}^{1 \circ} \wp_{i}^{-1}$ is a rotation with an angle $\alpha(i)$, where $\xi_{i}$ is an identification of the circle $\mathbf{S}_{i}^{1}$ to a reference circle $\mathbf{S}^{1}$.

We call these maps compositions of rotation in $n$ circles. They will also be denoted by $F_{7, \alpha}$.

Remark 3.1. The composition of two compositions of rotations in $n$ circles is again a composition of rotations in $n$ circles. More precisely, we have

$$
F_{\tau, \alpha^{\circ}} \circ F_{\tau^{\prime}, \alpha^{\prime}}=F_{\tau^{\circ} \tau^{\prime}, \alpha^{\prime}+\alpha^{\circ} \tau^{\prime}}
$$

Remark 3.2. If in the definition of a composition of rotations in $n$ circles we replace the assumption that $\tau$ is a permutation by the weaker assumption that $\tau$ is just a map, then it is easy to reduce this problem to the case in which $\tau$ is bijective. Moreover, since each permutation can be decomposed in a product of independent cyclic permutations, the case in which $\tau$ is a cyclic permutation is the one which is going to keep our attention in the sequel. However, the study of certain compositions of rotations in $n$ circles with $\tau$ not being a cyclic permutation (mainly the case $\tau=i d$ ) is still important and will be done later.

In the rest of the paper we are going to study the symbolic dynamics of this class of maps. In fact we shall look for the characterization of the kneading sequences associated to a natural coding and we shall describe some of their properties.

\section{SYMBOLIC DYNAMICS OF COMPOSITIONS OF ROTATIONS IN $\boldsymbol{n}$ CIRCLES}

We shall start by choosing a model to represent the compositions of rotations in $n$ circles. In what follows we shall denote by $E(\cdot)$ the integer part function. Let $\alpha$ be a map from a subset of $\mathbf{Z}$ to $\mathbf{R}$. We shall denote by $\bar{\alpha}$ the decimal part of $\alpha$ (i.e., $\vec{\alpha}=\alpha-E^{\circ} \alpha$ ).

We shall model a composition of rotations in $n$ circles $F_{\tau, \alpha}$ by a map $f_{\tau, \alpha}:[0, n) \rightarrow[0, n)$ defined in the following way. First, to a point $x$ of $[0, n)$, we shall associate its address $a(x)$ as follows:

$$
a(x)= \begin{cases}0, & \text { if } E(x) \leqslant x<E(x)+1-\bar{\alpha}(E(x)), \\ 1, & \text { if } E(x)+1-\bar{\alpha}(E(x)) \leqslant x<E(x)+1 .\end{cases}
$$

Then we set (see Fig. 10)

$$
f_{\tau, \alpha}(x)=\tau(E(x))+x-E(x)+\bar{\alpha}(E(x))-a(x) .
$$

Remark 4.1 We note that if $x \in[k, k+1)$ with $k \in\{0,1, \ldots, n-1\}$, then $f_{\tau, \alpha}(x) \in[\tau(k), \tau(k+1))$ and, if we 

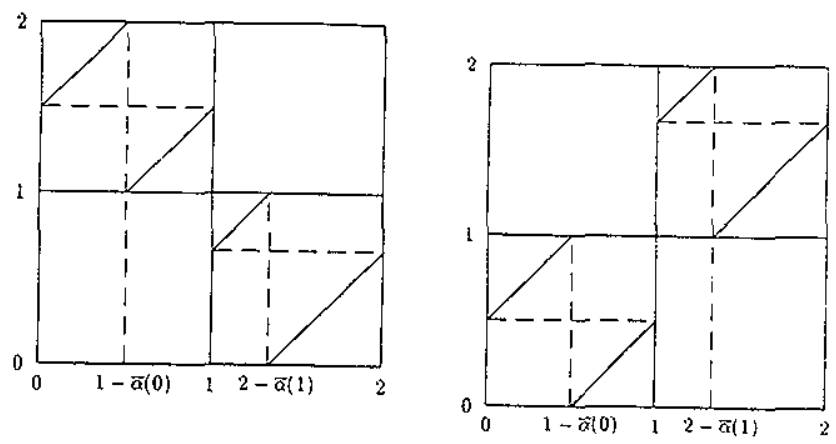

FIG. 10. Some compositions of rotations in two circles.

identify the circle $\mathbf{S}_{i}^{1}$ with the interval $[i, i+1)$ for each $i=0,1, \ldots, n-1$, then $\wp_{\tau(k)}{ }^{\circ} f_{\tau, \alpha^{\circ}} \wp_{k}^{-1}$ is a rotation by an angle $\alpha(k)$.

The itinerary of a point is then defined by the sequence

$$
\underline{I}(x)=E(x) a(x) a\left(f_{\tau, \alpha}(x)\right) a\left(f_{\tau, \alpha}^{2}(x)\right) \cdots \text {. }
$$

The $n$-tuple of itineraries $[\underline{I}(0), \underline{I}(1), \ldots, \underline{I}(n-1)]$ will be called the kneading invariant associated to $f_{\tau, \alpha}$. We note that all itineraries of the form $I(k)$ with $k \in\{0,1, \ldots, n-1\}$ start with $k 0 \ldots$. That is, they can be written in the form $\underline{I}(k)=k 0 d_{1} d_{2} \ldots$ for each $k=0,1, \ldots, n-1$.

We endow the space of itineraries with the following total ordering relation:

$$
s d_{0} d_{1} \cdots \cdots<s^{\prime} d_{0}^{\prime} d_{1}^{\prime} \cdots
$$

if either

$$
s<s^{\prime}
$$

or

$$
s=s^{\prime} \quad \text { and } d_{k}<d_{k}^{\prime},
$$

where $k \geqslant 0$ is such that $d_{i}=d_{i}^{\prime}$ for $i=0,1, \ldots, k-1$ and $<$ is the usual ordering of real numbers. Clearly, $x \leqslant y$ implies $\underline{I}(x) \leq \underline{I}(y)$.

Finally we define the shift operation $\sigma$ in the space of itineraries by

$$
\sigma\left(s d_{0} d_{1} \ldots\right)=\tau(s) d_{1} d_{2} \ldots .
$$

We note that the shift operation depends on $\tau$ and, therefore, on the map under consideration. In what follows we shall denote the composition $\sigma^{\circ} \sigma^{\circ} \cdots \circ \sigma$ ( $n$ times) by $\sigma^{n}$. As it is usual, for each $x \in[0, n)$ we have

$$
\sigma(\underline{I}(x))=\underline{I}\left(f_{\tau, \alpha}(x)\right) \text {. }
$$

The following proposition gives us an algorithm to compute the kneading invariant associated to $f_{\tau, \alpha}$.

Proposition 4.2. Let $f_{\tau, \alpha}$ be a composition of rotations in $n$ circles. Let $k \in\{0,1, \ldots, n-1\}$ and $\underline{I}(k)=k d_{0} d_{1} \ldots$ Then,

$$
\begin{aligned}
d_{l} & =E\left(\sum_{i=0}^{l} \bar{\alpha}\left(\tau^{i}(k)\right)\right)-E\left(\sum_{i=0}^{l-1} \bar{\alpha}\left(\tau^{i}(k)\right)\right) \text { for } l \\
& =0,1, \ldots
\end{aligned}
$$

Proof. From Remarks 4.1 and 3.1 we obtain by induction that

$$
f_{\tau, \alpha}^{l}=f_{\tau^{l}, \alpha+\alpha^{\circ} \tau+\alpha^{\circ} \tau^{2}+\cdots+\alpha^{\circ} \tau^{l-1}}
$$

for $l \geqslant 0$. Thus,

$$
f_{\tau, \alpha}^{l}(k)=\tau^{l}(k)+\sum_{i=0}^{l-1} \bar{\alpha}\left(\tau^{i}(k)\right)-E\left(\sum_{i=0}^{l-1} \bar{\alpha}\left(\tau^{i}(k)\right)\right) .
$$

Hence, $d_{l}=1$ if and only if

$$
\sum_{i=0}^{l-1} \bar{\alpha}\left(\tau^{i}(k)\right)-E\left(\sum_{i=0}^{l-1} \bar{\alpha}\left(\tau^{i}(k)\right)\right) \geqslant 1-\bar{\alpha}\left(\tau^{l}(k)\right) .
$$

Therefore,

$$
d_{l}=E\left(\sum_{i=0}^{l} \bar{\alpha}\left(\tau^{i}(k)\right)\right)-E\left(\sum_{i=0}^{l-1} \bar{\alpha}\left(\tau^{i}(k)\right)\right) .
$$

A first characterization of the kneading invariant of a composition of rotations in $n$ circles is given by the following proposition.

Proposition 4.3. Let $[I(0), \ldots, I(n-1)]$ be a kneading invariant of a composition of rotations in $n$ circles $f_{\tau, \alpha}$. Let $k \in\{0,1,2, \ldots, n-1\}$ and $j \geqslant 0$. Then we have

$$
\underline{I}\left(\tau^{j}(k)\right)=\tau^{j}(k) 0 d_{1} d_{2} \cdots \leqslant \sigma^{j}(\underline{l}(k)) \leqslant \tau^{j}(k) 1 d_{1} d_{2} \ldots .
$$

Proof. Since for each $x \in[0, n)$ we have $\sigma(I(x))$ $=\underline{I}\left(f_{\tau, \alpha}(x)\right)$ it follows that $\sigma^{j}(\underline{I}(k))=\underline{I}\left(f_{\tau, \alpha}^{j}(k)\right)$ $=\underline{I}\left(f_{\tau^{j}, \alpha+\alpha \circ \tau+\alpha^{\circ} \tau^{2}+\cdots+\alpha^{\circ} \tau^{j-1}}(k)\right)$. Therefore, $\quad f_{\tau, \alpha}^{j}(k)$ $\in\left[\tau^{j}(k), \tau^{j}(k)+1\right)$. On the other hand, if $x \in[l, l+1)$ we have that $\underline{I}(l)=l 0 d_{1} d_{2} \ldots \leqslant \underline{I}(x) \leqslant l 1 d_{1} d_{2} \ldots$. This ends the proof of the proposition.

We are interested in giving a full characterization of the kneading invariants of the compositions of rotations in $n$ circles. To do this, in addition to the above two properties, a third condition is necessary. It turns out that this last condition is strongly related to the characterization of the kneading sequences of rotations. Therefore, we shall study first this particular case. This is the subject of the next section.

\section{ROTATIONS}

The main results in this section are closely related to the ones developed by Morse and Hedlund in Ref. 5 when studying Sturmian series and follow the ones from Gambaudo (see Ref. 1) with few improvements. We are going to characterize the kneading invariants of rotations by means of some structural properties among which the lexicographical ordering of 
sequences plays a fundamental role. However, in Ref. 5, different motivation can be found. In fact, they study the relation between rotations and all associated symbolic sequences (not only kneading sequences) through the rotation number. Therefore, because of these differences in motivation and approach with Ref. 5, and also for completeness we shall develop our own study in full detail.

A rotation can be modeled as a composition of rotations in $n$ circles in the trivial case in which $n=1$. Then, of course, $\tau \equiv i d$. We note that in the case $n>1$ and $\tau \equiv i d$ we have $n$ noncoupled rotations and, hence, the characterization of the kneading invariant in this case follows directly from the corresponding characterization for rotations.

When we consider a rotation as a composition of rotations in one circle the use of $\tau$ is superfluous and, hence, it will be omitted. Also, $\alpha(0)$ will be denoted simply by $\alpha$. Moreover, the use of the first symbol in the itinerary of a point is aiso superfluous and so it will be removed. Thus, when talking about rotations, the itinerary $\underline{I}(x)$ of a point will be defined to be the sequence

$$
a(x) a\left(f_{\alpha}(x)\right) a\left(f_{\alpha}^{2}(x)\right) \cdots
$$

We extend the notions of kneading invariant, ordering of itineraries, and shift operation to this new framework in the natural way.

From all that was mentioned in the previous section it follows that if $\underline{I}=d_{0} d_{1} \ldots$ is the kneading invariant of a rotation, then it satisfies the following two conditions:

(A) $d_{0}=0$

(B) $\underline{I} \leqslant \sigma^{j}(I) \leqslant 1 d_{1} d_{2} \ldots$ for all $j \geqslant 0$.

We want to see that each kneading invariant of a rotation satisfies one more condition. This third condition, together with conditions (A) and (B), characterizes the kneading invariants of rotations. This will be shown at the end of this section. To state the property of rotations we are looking for, we need some definitions and technical lemmas.

An infinite sequence of 0 's and 1 's will be called admissible. If, in addition, it satisfies properties (A) and (B) then it will be called extremal.

Lemma 5.1. Let $I=d_{0} d_{1} d_{2} \ldots$ be extremal and let $1 \leqslant j<k$ be such that $d_{1} \ldots d_{j-1}=d_{k-j+1} \ldots d_{k-1}$ and $d_{j} \neq d_{k}$. Then $d_{k-j}=d_{j}$.

Proof. Since $\underline{I}$ is extremal we have

$$
\begin{aligned}
\underline{I} & =0 d_{1} \ldots d_{j-1} d_{j} \ldots \leqslant \sigma^{k-j}(\underline{I}) \\
& =d_{k-j} d_{k-j+1} \ldots d_{k-1} d_{k} \ldots \leqslant 1 d_{1} \ldots d_{j-1} d_{j} \ldots .
\end{aligned}
$$

If $d_{j}=1$ and $d_{k}=0$ then, from the first inequality it follows that $d_{k-j}=1=d_{j}$. If $d_{j}=0$ and $d_{k}=1$ then, from the second inequality we obtain $d_{k-j}=0=d_{j}$.

Proposition 5.2. Let $I$ be an extremal sequence different from $0^{\infty}$ and $01^{\infty}$. Then there exists $b \geqslant 1$ such that $\underline{I}$ is either $01^{b} 01^{b} 101^{b_{2}} 0 \ldots$ or $0^{b+1} 10^{b_{1}} 10^{b_{2}} 1 \ldots \quad$ with $b_{i} \in\{b, b+1\}$ for all $i \geqslant 1$.

Proof. Let $I=0 d_{1} d_{2} \ldots$. By using Lemma 5.1 with $j=1$ we get $d_{k-1}=\bar{d}_{1}$ for all $k>1$ such that $d_{k} \neq d_{1}$. That is, if $d_{1}=0$ then in the sequence $I$ there always is a 0 before any 1 and if $d_{1}=1$ then there is a 1 before any 0 . Then, $I$ is either $01^{b_{11}} 01^{b_{1}} 01^{b_{2}} 0 \ldots$ or $0^{b_{1}} 10^{b_{1}} 10^{b_{2}} \ldots$ where $b_{i} \geqslant 1$ for all $i \geqslant 0$. Assume that $\underline{I}$ is of the form $0^{b_{0}} 10^{b_{1}} 10^{b_{2}} \ldots$. The proof in the other case follows similarly.

Since $I$ is extremal, for $i \geqslant 0$ we get $0^{b_{1}} 10^{b_{1}} 1 \ldots$ $\leqslant \sigma^{m_{i}}(\underline{I})=0^{b_{i}} 10^{b_{i+1}} 1 \ldots \leqslant 10^{b_{0}-1} 10^{b_{1}} 1 \ldots$ with $m_{i}=i$ $+b_{0}+b_{1}+\cdots b_{i-1}$. Hence, $b_{i} \leqslant b_{0}$. Similarly, $0^{b_{11}} 10^{b_{1}} 1 \ldots$ $\leqslant \sigma^{m_{i}-1}(I)=10^{b_{i}} 10^{b_{i+1}} 1 \ldots \leqslant 10^{b_{0}-1} 10^{b_{1}} 1 \ldots$ and hence, $b_{i} \geqslant b_{0}-1$. If $b_{0}>1$ we set $b=b_{0}-1$ and $\underline{I}$ $=0^{b+1} 10^{b_{1}} 110^{b_{2}} 21 \ldots$ with $b_{i} \in\{b, b+1\}$ for all $i \geqslant 0$. If $b_{0}=1$ then we set $b=1$ and we get $I=010101 \ldots$ $=01^{b} 01^{b} 101^{b_{2}} \ldots$ with $b_{i}=b$ for all $i \geqslant 1$. This ends the proof of the proposition.

The above proposition motivates the next definition. Let $\underline{I}$ be an admissible sequence. We shall say that $\underline{I}$ is 0 -nice (respectively, 1-nice) if there exists $b \geqslant 1$ such that $\underline{I}=0^{b_{1}} 10^{b_{1}} 10^{b_{2}} 1 \ldots \quad$ (respectively, $\underline{I}=01^{b_{0}} 01^{b_{1}}{ }_{0} 01^{b_{2}} 0 \ldots$ ) with $b_{i} \in\{b, b+1\}$ for all $i \geqslant 0$ and $b_{i}=b+1$ for some $i$. The number $b$ will be called the order of $\underline{I}$. A nice sequence will be a sequence either 0 -nice or 1 -nice or equals to $(01)^{\infty}$ or $0^{\infty}$ or $01^{x}$.

Remark 5.3. Notice that each sequence of the form $\left(01^{k}\right)^{\%}$ with $k>1$ is 1 -nice of order $k-1$. Therefore, in view of Proposition 5.2, each extremal sequence is nice. However, as we shall see later, there exist nice sequences which are not extremal.

Now we define the deflation operation $\delta$ from the space of nice sequences to the space of admissible ones. First we set $\delta\left((01)^{\infty}\right)=\delta\left(0^{\infty}\right)=00^{\infty}$ and $\delta\left(01^{\infty}\right)=01^{\infty}$. Next we define $\delta$ on the rest of nice sequences. Let $\underline{I}$ be a $t$-nice sequence of order $b$. If $t=1$ and $I=A_{0} A_{1} A_{2} \ldots$ with $A_{i}=01^{b_{i}}$ for $i \geqslant 0$, then we set $\delta(I)=B_{0} B_{1} B_{2} \ldots$, where

$$
B_{i}=\left\{\begin{array}{l}
0, \quad \text { if } A_{i}=01^{b}, \\
01, \quad \text { if } A_{i}=01^{b+1},
\end{array}\right.
$$

for $i \geqslant 0$. If $t=0$ and $\underline{I}=A_{0} A_{1} A_{2} \ldots$ with $A_{0}=0^{b_{11}}$ and $A_{i}$ $=10^{b_{i}}$ for $i \geqslant 1$, then $\delta(I)=B_{0} B_{1} B_{2} \ldots$ where $B_{0}=1-b_{0}+b$ and

$$
B_{i}=\left\{\begin{array}{l}
1, \quad \text { if } A_{i}=10^{h}, \\
10, \quad \text { if } A_{i}=10^{b+1},
\end{array}\right.
$$

for $i \geqslant 1$.

We shall study now the action of $\delta$ on the set of extremal sequences. We want to show that $\delta$ preserves the extremality and, therefore, it can be iterated infinitely many times on the set of extremal sequences. This will be done in Proposition 5.6. Prior to the proof of this fact we shall see that the deflation operation preserves ordering and, in some sense, commutes with $\sigma$. In the rest of the section we shall use freely the notation from the definitions of a nice sequence and $\delta$.

Lemma 5.4. Let $\underline{I}<\underline{J}$ be t-nice sequences of the same order with $t \in\{0,1\}$. Then $\delta(I)<\delta(J)$.

Proof. We shall prove the lemma in the case $t=0$. The proof for the case $t=1$ follows similarly. Let $\underline{I}=0^{b_{0}} 10^{b_{1}} 10^{b_{2}} 1 \ldots$ and $\underline{J}=0^{k_{0}} 10^{k_{1}} 10^{k_{2}} 1 \ldots$, and assume that $\underline{I}$ and $\underline{J}$ have order $b$. Since $\underline{I}<\underline{J}$ there exists $l \geqslant 0$ such that $b_{l} \neq k_{l}$ and $b_{i}=k_{i}$ for $i=0,1, \ldots, l-1$. Clearly, $b_{l}>k_{l}$. Therefore, $b_{l}=b+1$ and $k_{l}=b$. If $l=0$ then 
the lemma follows directly from the definition of $\delta$. If $l>0$ we get $\delta(I)=\left(1-b_{0}+b\right)_{\tilde{1}} B_{1} B_{2} \ldots B_{l-1} B_{l} B_{l+1} \ldots$ and $\delta(J)=\left(1-b_{0}+b\right) B_{1} B_{2} \ldots B_{l-1} \tilde{B}_{l} B_{l+1} \ldots$ with $B_{l}=10$ and $\tilde{B}_{l}=1$. Therefore, $\delta(\underline{I})<\delta(\underline{J})$ because $\tilde{B}_{l+1}$ is either 1 or 10 .

Lemma 5.5. Let $I$ be an extremal t-nice sequence of order $b$ with $t \in\{0,1\}$ such that $\delta(I) \neq 01^{\infty}$. Let $m \geqslant 0$ and $l$ be such that $\sigma^{m}(\delta(I))=a B_{l+1} B_{l+2} \ldots$ where $a \in\{0,1\}$. If $r=l+b_{0}+b_{1}+\cdots+b_{l-1}$, then $\sigma^{r}(\underline{I})$ is also $t$-nice of order $b$ and $\delta\left(\sigma^{r}(I)\right)=\sigma^{m}(\delta(\underline{I}))$.

Proof. If $m=0$ then since $I$ is extremal and $t$-nice it follows that $B_{0}=0=a$ and $l=0$. So, $r=0$ and there is nothing to prove. Now assume that $m>0$. We also assume $t=0$ and $a=1$. In the other three cases the proof follows similarly. Clearly, $\sigma^{r}(I)=0^{b} 10^{b_{1+1}} 1 \ldots$ with $b_{i} \in\{b, b+1\}$ for all $i \geqslant l$. Since $a=1$ and $t=0$, in view of the definition of $\delta$, it follows that $B_{l}=1$ and $b_{l}=b$. We claim that $b_{i}=b+1$ for some $i>l$ [and hence, $\sigma^{\prime}(I)$ is 0 -nice]. Otherwise, $b_{i}=b$ for $i \geqslant l$. Since $I$ is 0 -nice, in view of Proposition 5.2 we have $\underline{I}=0^{b_{0}+1} 10^{b_{1}} 10^{b_{2}} 1 \ldots$. Moreover, since $\delta(I) \neq 01^{\curvearrowright}$ there exists $b_{j}$ with $j \geqslant 1$ such that $b_{j}=b+1$ and $b_{i}=b$ for $i=1,2, \ldots, j-1$. Therefore,

$$
\begin{aligned}
& 10^{b} 10^{b_{1}} 10^{b_{2}} \ldots 10^{b_{j}} 1 \ldots \\
& =10^{b} 10^{b} 10^{b} \ldots 10^{b+1} 1 \ldots<\left(10^{b}\right)^{\infty}=\sigma^{r+b}(\underline{I}),
\end{aligned}
$$

which contradicts the extremality of $\underline{I}$. This ends the proof of the claim.

Since $\sigma^{r}(\underline{I})$ is 0-nice we obtain

$$
\begin{aligned}
\delta\left(\sigma^{r}(\underline{I})\right) & =\delta\left(0^{b} 10^{b_{l}+1} 10^{b_{l}+2} \ldots\right)=1 B_{l+1} B_{l+2} \ldots \\
& =\sigma^{m}(\delta(\underline{I}))
\end{aligned}
$$

Proposition 5.6. If $\underline{I}$ is extremal, then $\delta(\underline{I})$ is defined and extremal.

Proof. If $I \in\left\{(01)^{\infty}, 0^{\infty}, 01^{\infty}\right\}$ then there is nothing to prove. Thus, we assume that $\underline{I}\left\{(01)^{\infty}, 0^{\infty}, 01^{\infty}\right\}$. By Proposition 5.2 and Remark $5.3, I$ is $t$-nice with $t \in\{0,1\}$. Assume that $I$ is 0 -nice. If $I$ is 1 -nice the proof follows analogously.

If $\delta(\underline{I})=01^{\infty}$ the proposition holds trivially. So, we also assume that $\delta(I) \neq 01^{\infty}$. In view of Proposition 5.2, $\underline{I}$ $=0^{b+1} 10^{b} 110^{b} 21 \ldots$ with $b_{i} \in\{b, b+1\}$ for all $i \geqslant 1$ and $b \geqslant 1$. From the definition of $\delta$ we get $\delta(\underline{I})=0 B_{1} B_{2} \ldots$ with $B_{i} \in\{1,10\}$ for all $i \geqslant 1$. Hence, condition (A) of extremality is satisfied. Now we prove condition $(B)$.

Let $m>0$. If $\sigma^{m}(\delta(\underline{I}))=B_{l} B_{l+1} \ldots$ for some $l \geqslant 1$ then $\delta(\underline{I})<\sigma^{m}(\delta(I))$. We have to see that $\sigma^{m}(\delta(I)) \leqslant 1 B_{1} B_{2} \ldots$ If $B_{l}=10$ this holds because $B_{1}$ starts with 1 . So, assume $B_{l}=1$. In view of Lemma $5.5, \sigma^{r}(I)$ is 0 -nice of order $b$ and $\sigma^{m}(\delta(\underline{I}))=\delta\left(\sigma^{r}(\underline{I})\right)$ where $r=l+b_{0}+b_{1}+\ldots+b_{l-1}$. We note that $\sigma^{r}(I)=0^{b} 10^{b_{l+1}} 1 \ldots=0^{b} 10^{b_{l+1}} \ldots$ because $B_{l}=1$. Let us denote by $J$ the sequence $0^{b} 10^{b_{1}} 10^{b_{2}} \ldots$. Since $\delta(I) \neq 01^{\infty}$ we get that $I \neq 0^{b+1}\left(10^{b}\right)^{\infty}$ and, hence, $\underline{J} \neq\left(0^{b} 1\right)^{\infty}$. Therefore, $J$ is 0 -nice of order $b$ and, thus, $\delta(J)=1 B_{1} B_{2} \ldots$. Since $l \geqslant 1$ and $b_{0} \in\{b, b+1\}$ we get $r \geqslant b+1$. So, by the extremality of $\underline{I}$, we have $\sigma^{r-1}(\underline{I})$ $=10^{b} 10^{b_{l+1}} 1 \ldots \leqslant 10^{b} 10^{b} 110^{b_{2}} \ldots$. Hence, $\sigma^{r}(\underline{I}) \leqslant \underline{J}$ and, by Lemma $5.4, \quad \delta\left(\sigma^{r}(\underline{I})\right) \leqslant \delta(J)$. In short, $\sigma^{m}(\delta(I)) \leqslant 1 B_{1} B_{2} \ldots$.
Now assume that $\sigma^{m}(\delta(I))=0 B_{1+1} B_{I+2} \ldots$ for some $l \geqslant 1$ (that is, $B_{l}=10$ ). Clearly, $\sigma^{m}(\delta(I))<1 B_{1} B_{2} \ldots$. On the other hand, again by Lemmas 5.4 and 5.5 and by the extremality of $\underline{I}$, we get that

$$
\delta(\underline{I}) \leqslant \delta\left(\sigma^{r}(\underline{I})\right)=\sigma^{m}(\delta(\underline{I})),
$$

where $r$ is as in Lemma 5.5 .

Now we start looking at the properties of the kneading invariants of rotations. We shall use the following notation,

$$
\tilde{E}(x)= \begin{cases}E(x), & \text { if } x \notin \mathbf{Z}, \\ x-1, & \text { if } x \in \mathbf{Z} .\end{cases}
$$

From Sec. II.2.2 of Ref. 1 with the appropriate changes of notation we have the following result which gives a geometric interpretation of the deffation operation on the kneading invariants.

Proposition 5.7. Let $\underline{I}$ be the kneading invariant of the rotation by angle $\alpha$. Set $\gamma=\alpha /(1-\alpha)$. Then the following statements hold.

(1) If $\alpha<1 / 2$ then $I$ is 0 -nice and $\delta(\underline{I})$ is the kneading invariant of the rotation by angle

$$
\beta=\frac{1}{\gamma^{-1}-\tilde{E}\left(\gamma^{-1}\right)+1} .
$$

(2) If $\alpha>1 / 2$ then $I$ is 1 -nice and $\delta(I)$ is the kneading invariant of the rotation by angle

$$
\beta=\frac{\gamma-\tilde{E}(\gamma)}{\gamma-\tilde{E}(\gamma)+1} .
$$

(3) If $\alpha=1 / 2$ then $I=(01)^{\infty}$ and $\delta(\underline{I})=0^{\infty}$ is the kneading invariant of the rotation by angle $\beta=0$.

Conversely, if $I=a_{0} a_{1} \ldots \neq 0^{\infty}$ is the kneading invariant of the rotation by angle $\beta$ and $k \in \mathbf{N}$ then the new sequence obtained by applying to $\underline{I}$ the rule

$$
a_{i} \rightarrow\left\{\begin{array}{l}
0^{k+1}, \quad \text { if } i=0 \\
10^{k}, \quad \text { if } i>0 \text { and } a_{i}=1 \\
0, \quad \text { if } i>0 \text { and } a_{i}=0
\end{array}\right.
$$

or, respectively,

$$
a_{i} \rightarrow\left\{\begin{array}{l}
01^{k}, \quad \text { if } a_{i}=0, \\
1, \quad \text { if } a_{i}=1,
\end{array}\right.
$$

is the kneading invariant of the rotation by angle $\alpha$ where $\alpha$ satisfies the relation give in (1) and $k=\tilde{E}[(1-\alpha) / \alpha]$ (respectively, the relation given in (2) and $k=\tilde{E}[\alpha /(1-\alpha)])$.

The following lemma shows that the kneading invariants of rotations cannot be any extremal sequence.

Lemma 5.8. Let $I$ be the kneading invariant of a rotation. Then, $I \neq 01^{\infty}$.

Proof. Assume that $\underline{I}$ is the kneading invariant of the rotation with angle $\alpha$. Without loss of generality we may assume that $\alpha \in[0,1)$. The statement $\underline{I}=01^{\infty}$ is equivalent to $a(k \alpha)=1$ for all $k \geqslant 1$ and, by the definition of address, this is equivalent to the condition $k \alpha-E(k \alpha) \geqslant 1-\alpha$ for all 
$k \geqslant 1$. If $\alpha$ is rational, say $p / q$, then taking $k=q$ we get $k \alpha-E(k \alpha)=0<1-\alpha$. If $\alpha$ is irrational then the sequence $\{k \alpha-E(k \alpha)\}_{k \in \mathrm{N}}$ is dense in $[0,1)$. Thus, again there exists $k \geqslant 1$ such that $k \alpha-E(k \alpha)<1-\alpha$. hence, $\underline{l} \neq 01^{*}$.

By Proposition 5.7 and Lemma 5.8 we see that if $I$ is the kneading invariant of a rotation, then $\delta^{n}(\underline{I}) \neq 01^{\infty}$ for all $m \geqslant 0$. Therefore, it is interesting to characterize the set of extremal sequences having some iterate by $\delta$ equal to $01 \%$. This is our next step.

Let $I$ be an admissible sequence. We shall say that $I$ is periodic if it exists $p>0$ such that $\sigma^{p}(\underline{I})=\underline{I}$. The smallest such $p$ will be called the period of $I$ (in this case $I$ will also be called $p$-periodic). The sequence $I$ is $p$-eventually periodic if there exists $m \geqslant 0$ such that $\sigma^{m}(I)$ is periodic of period $p$. The $p$-eventually periodic sequences which are not periodic will be called p-preperiodic (or simply preperiodic). If $A$ is a finite sequence then $|\underline{A}|$ will denote the cardinality of $\underline{A}$.

Lemma 5.9. The only extremal 1-preperiodic sequence is $01^{\circ}$.

Proof. Let $I$ be extremal 1-preperiodic. Then $I=0 d_{1} d_{2} \ldots d_{m-1}\left(d_{m}\right)^{x}$ with $m>0$. If $d_{m}=0$, by the extremality $\underline{I} \leqslant \sigma^{m}(I)=0^{\infty}$. Hence, $\underline{I}=0^{\infty}$ which is periodic, a contradiction. If $d_{m}=1$ then $\sigma^{m}(\underline{I})=1^{\infty} \leqslant 1 d_{1} d_{2} \ldots d_{m-1} 1^{\%}$. Hence, $d_{1}=d_{2}=\cdots=d_{m-1}=1$ and then, $\underline{I}=01^{x}$.

Lemma 5.10. Let $\underline{I}$ be extremal and p-preperiodic (respectively, $p$-periodic) with $p>1$. Then $\delta(I)$ is $q$-preperiodic (respectively, $q$-periodic) with $q<p$. Moreover, if $\underline{I}$ is not eventually periodic then $\delta(\underline{I})$ is not eventually periodic.

Proof. In view of Proposition 5.6, $\delta(\underline{I})$ is well defined because $I$ is extremal. Assume that $I$ is $p$-preperiodic with $p>1$. We have that $I \notin\left\{0^{\infty},(01)^{\varkappa}, 01^{\%}\right\}$. In view of Proposition $5.2, I$ is $t$-nice with $t \in\{0,1\}$ because $I$ is extremal. Assume that $\underline{I}$ is 1 -nice of order $b$ with $b \geqslant 1$. The case in which $I$ is 0 -nice follows similarly.

Since $I$ is $p$-preperiodic it can be written as $A_{0} A_{1} \ldots A_{k n-1}\left(A_{k n} \ldots A_{(k+1) n-1}\right)^{\infty}$ with $A_{i} \in\left\{01^{b}, 01^{b+1}\right\}$ for $i \geqslant 0,\left|A_{k n} \ldots A_{(k+1) n-1}\right|=p$ and $k \geqslant 1$ is the smallest integer such that $A_{(k-1) n} \ldots A_{k n-1} \neq A_{k n} \ldots A_{(k+1) n-1}$. Then $\delta(\underline{I})=B_{0} B_{1} \ldots B_{k n-1}\left(B_{k n} \ldots B_{(k+1) n-1}\right)^{x}$ with $B_{(k-1) n} \ldots$ $B_{k n-1} \neq B_{k n} \ldots B_{(k+1) n-1}$. Therefore, $\delta(I)$ is $q$-preperiodic for some $q \geqslant 1$. In view of the definition of $\delta$ we have $\left|B_{i}\right|<\left|A_{i}\right|$ for all $i \geqslant 0$. Hence, $q<p$.

If $I$ is $p$-periodic with $p>1$ then $I \notin\left\{0^{\infty}, 01^{\infty}\right\}$. If $\underline{I}=(01)^{x}$ then $\delta(\underline{I})=0^{x}$ and the proposition follows. Hence, we assume that $\underline{I} \notin\left\{0^{\infty},(01)^{\infty}, 01^{\infty}\right\}$. Then the lemma follows as in the preperiodic case.

Finally, in order that $\delta(\underline{I})$ be eventually periodic, from the definition of $\delta$, it follows that $I$ has to be eventually periodic too. This ends the proof of the lemma.

Lemma 5.11. Let $I$ be extremal such that $\delta(I)=0\left(B_{1} B_{2} \ldots B_{k}\right)^{\infty}$ with $k \geqslant 1, B_{i} \in\{0,01,1,10\}$ for $i=1,2, \ldots, k-1$ and $B_{k} \in\{01,1\}$. Then $\underline{I}=0\left(\tilde{B}_{1} \tilde{B}_{2} \ldots \tilde{B}_{l}\right)^{\infty}$ with $l \geqslant 1, \tilde{B}_{i} \in\{0,01,1,10\}$ for $i=1,2, \ldots, l-1$ and $\tilde{B}_{l} \in\{01,1\}$. In particular, $I$ is p-preperiodic for some $p \geqslant 1$.

Proof. Since $\delta(\underline{I}) \neq 0^{\infty}$ we have that $\underline{I} \notin\left\{0^{\infty},(01)^{\infty}\right\}$. If $\underline{I}=01^{\infty}$ then the lemma follows. Hence, we may assume $I$ is $t$-nice of order $b \geqslant 1$ with $t \in\{0,1\}$ because it is extremal.
We shall prove the lemma in the case $t=1$. The case $t=0$ follows similarly. By the definition of $\delta, I$ can be written as $01^{b}\left(A_{1} \ldots A_{k-1} 01^{b+1}\right)^{\infty}$ with $A_{i}=01^{b_{i}}$ and $b_{i} \in\{b, b+1\}$ for $i=1,2, \ldots, k-1$. Thus,

$$
\begin{aligned}
I & =0\left(1^{b} A_{1} \ldots A_{k-1} 01\right)^{\infty}=0\left(1^{b} 01^{b} 101^{b_{2}} \ldots 01^{b_{k-1}} 01\right)^{\infty} \\
& =0\left(1^{b-1} 101^{b_{1}-1} 10 \ldots 1^{b_{k-1}-1} 101\right)^{\infty} .
\end{aligned}
$$

The next proposition characterizes the extremal sequences which have some iterate by $\delta$ equal to $01^{\circ}$.

Proposition 5.12. An extremal sequence I satisfies $\delta^{\prime \prime}(I)=01^{\curvearrowright}$ for some $m \geqslant 0$ if and only if $\underline{I}$ is p-preperiodic for some $p \geqslant 1$.

Proof. Assume first that $\underline{I}$ is $p$-preperiodic with $p \geqslant 1$. If $p=1$ then, by Lemma $5.9, \underline{I}=01^{\circ}$. Assume that $p>1$. By Lemma 5.10 and Proposition 5.6, $\delta(I)$ is extremal and $q$-preperiodic with $q<p$. By iterating this process we obtain that $\delta^{\prime \prime}(\underline{I})=01^{\infty}$ for some $m>0$.

Now suppose that $\delta^{n}(I)=01^{\infty}$ for some $m \geqslant 0$. Then the proposition follows directly from the inductive use of Lemma 5.11.

We note that, from Proposition 5.12 and the iterative use of Lemma 5.11 we get that each extremal $p$-preperiodic sequence is of the form $O(\underline{A} 1)^{x}$ with $\underline{A}$ a finite sequence of 0 's and 1's.

From Proposition 4.3 we see that each kneading invariant of a rotation $\underline{I}$ is extremal. By Proposition 5.7 we get that $\delta^{\prime \prime}(I)$ is a kneading invariant of a rotation for each $m \geqslant 0$. Therefore, by Lemma $5.8, \delta^{\prime \prime}(\underline{I}) \neq 01^{\infty}$ for each $m \geqslant 0$. So, $I$ is not preperiodic by Proposition 5.12. Next we show that indeed the extremality and the non-preperiodicity of a sequence characterize the kneading invariants of rotations.

Theorem 5.13. An admissible sequence $I$ is the kneading invariant of a rotation if and only if is extremal and not preperiodic.

Proof. The "only if" part follows from Propositions 5.7 and 5.12 and Lemma 5.8.

Now we prove that if $\underline{I}$ is extremal and not preperiodic then it is the kneading invariant of a rotation. Let us split the study into two cases.

Case $1 . I$ is periodic. In view of Lemma 5.10 , by iterating the deflation process we eventually get

$$
\delta^{n}(I)=\delta_{n} \circ \delta_{n-1} \circ \cdots \circ \delta_{2} \circ \delta_{1}(I)=0^{\infty}
$$

for some $n \geqslant 0$ (where $\delta_{i}$ is the deflation transformation used at the step (i) for $i=1,2, \ldots, n$ ). Obviously, $0^{\infty}$ is the kneading invariant of the rotation with angle 0 . Using Proposition 5.7 we can reverse the above deflation process to get successively that $\delta_{n}^{-1}\left(0^{\infty}\right)$, $\delta_{n-1}^{-1} \circ \delta_{n}^{-1}\left(0^{\infty}\right), \ldots, \delta_{1}^{-1} \circ \cdots \circ \delta_{n}^{-1}\left(0^{\infty}\right)=\underline{I}$ are kneading invariants of rotations.

Case 2. $I$ is not eventually periodic. In view of Proposition 5.6 and Lemma 5.10 we can apply the deflation process infinitely many times without reaching a periodic sequence. Let $\delta_{1}, \ldots, \delta_{n}, \ldots$ be this sequence of deflations. Set $\underline{I}_{n}=\delta_{1}^{-1} \circ \delta_{2}^{-1} \circ \cdots \circ \delta_{n}^{-1}\left((01)^{\infty}\right)$ for $n \geqslant 1$. Then $\underline{I}_{n}$ is the kneading invariant of a rotation with rational angle $\alpha_{n}=p_{n} / q_{n}$ with $\left(p_{n}, q_{n}\right)=1$. By using Proposition 5.7 one can check 
(see the proof of Theorem II 2.2 .1 of Ref. 1) that the sequence $\left\{q_{n}\right\}_{n \in \mathrm{N}}$ is increasing and going to infinity with $n$. Furthermore,

$$
\left|\alpha_{n}-\alpha_{n+1}\right| \leqslant 1 / q_{n}^{2}
$$

for each $n$. Therefore, the sequence $\left\{\alpha_{n}\right\}$ converges to some value $\alpha^{*}$. The map which associates the angle of a rotation to the kneading invariant of the rotation is continuous when equipping the set of admissible sequences with the usual metric

$$
d\left(\left\{x_{n}\right\}_{n \in \mathrm{N}},\left\{y_{n}\right\}_{n \in \mathrm{N}}\right)=\sum_{n=0}^{\infty} \frac{\left|x_{n}-y_{n}\right|}{2^{n}} .
$$

Consequently, the sequence $\left\{\underline{I}_{n}\right\}_{n \in \mathrm{N}}$ of kneading invariants of rotations by angle $\alpha_{n}$ converges to the sequence $I$ which is the kneading invariant of the rotation by angle $\alpha^{*}$.

\section{EXTREMAL BLOCKS}

From the results obtained in Section IV (see Propositions 4.2 and 4.3$)$ we know that if $[\underline{I}(0), \ldots, \underline{I}(n-1)]$ is a kneading invariant of a composition of rotations in $n$-circles, then

(a) $\underline{I}(k)=k 0 d_{1} d_{2} \ldots$, and

(b) $I\left(\tau^{j}(k)\right)=\tau^{j}(k) 0 d_{1} d_{2} \ldots \leqslant \sigma^{j}(I(k))$

$\leqslant \tau^{j}(k) 1 d_{1} d_{2} \ldots$ for each $k=0, \ldots, n-1$ and $j \geqslant 0$.

Let $\left[0 \underline{A}_{0}, \ldots,(n-1) A_{n-1}\right]$ be a set of symbolic sequences, where $A_{i}$ are admissible sequences (in the sense of Sec. V), and let $\tau$ be a cyclic permutation of $\{0, \ldots, n-1\}$. We say that $\left[0 \underline{A}_{0}, \ldots,(n-1) \underline{A}_{n-1}\right]$ is an $n$-extremal block if

(An) $k A_{k}=k 0 d_{1} d_{2} \ldots$, and

(Bn) $\tau^{j}(k) A_{\tau^{j}(k)}=\tau^{j}(k) 0 d_{1} d_{2} \ldots \leqslant \sigma^{j}\left(k A_{k}\right)$ $\leqslant \tau^{j}(k) 1 d_{1} d_{2} \ldots$ for each $k=0, \ldots, n-1$ and $j \geqslant 0$.

Notice that these conditions correspond to conditions (a) and (b), and are the analogous to conditions (A) and (B) from Sec. $V$ for rotations.

Given an admissible sequence $\underline{A}=d_{0} d_{1} \ldots$ we say that $\underline{A}$ is weightable of order $m$ if $\left|d_{i} d_{i+1} \ldots d_{i+m-1}\right|_{1}$ $\in\left\{\left|0 d_{1} \ldots d_{m-1}\right|_{1},\left|1 d_{1} \ldots d_{m-1}\right|_{1}\right\}$, for all $i \geqslant 0$, where $\left|d_{i} \ldots d_{i+r}\right|_{1}$ denotes the number of elements $d_{j}$ equal to 1 , with $i \leqslant j \leqslant i+r$. That is, $\left.\mid d_{i} \ldots d_{i+r}\right\}_{1}=\sum_{j=0}^{r} d_{i+j}$.

If $A$ is weightable of order $m$, we define the weight of order $m$ of $A$, denoted by $\omega_{m}(\underline{A})$, as $\left|0 d_{1} \ldots d_{m-1}\right|_{1}$.

Next we will be interested in proving that all the sequences $A_{i}$ from an $n$-extremal block are weightable of order $n$. To do it, we need some auxiliary lemmas.

Lemma 6.1. Let $\left[0 \underline{A}_{0}, \ldots,(n-1) \underline{A}_{n-1}\right]$ be an $n$-extremal block with cyclic permutation $\tau$, and let $m, k, j$ and $j^{\prime}$ be integers such that $m, k \in\{0, \ldots, n-1\}$, $j, j^{\prime} \geqslant 0, \quad \tau^{j}(k)=\tau^{j^{\prime}}(m)=l, \quad \sigma^{j}\left(k \underline{A}_{k}\right)=l d_{0} d_{1} \ldots \quad$ and $\sigma^{j^{\prime}}\left(m A_{m}\right)=l d_{0}^{\prime} d_{1}^{\prime} \ldots$. Then the following hold:

(1) If $d_{0}=d_{1}=0$, then $d_{0}^{\prime} \neq 1$ or $d_{1}^{\prime} \neq 1$.

(2) If $d_{0}=d_{0}^{\prime}$ and $\sigma^{j}\left(k \underline{A}_{k}\right)<\sigma^{j^{\prime}}\left(m A_{m}\right)$, then $\sigma^{j+1}\left(k \underline{A}_{k}\right)<\sigma^{j^{\prime}+1}\left(m \underline{A}_{m}\right)$.

(3) If $d_{0} \neq d_{0}^{\prime}$ and $\sigma^{j}\left(k \underline{A}_{k}\right)<\sigma^{j^{\prime}}\left(m \underline{A}_{m}\right)$, then $\sigma^{j+1}\left(k A_{k}\right) \geqslant \sigma^{j^{\prime}+1}\left(m A_{m}\right)$.

Proof. (1) If $d_{0}=d_{1}=0$, then from the definition of an $n$-extremal block it follows that $l A_{l}=l 0 d_{1}^{\prime \prime} \ldots$ $\leqslant \sigma^{j}\left(k A_{k}\right)=l 00 d_{2} \ldots$ and so $d_{1}^{\prime \prime}=0$. Analogously, we have $l 00 \ldots \leqslant l d_{0}^{\prime} d_{1}^{\prime} \ldots \leqslant l 10 \ldots$. Therefore it is not possible that $d_{0}^{\prime}=d_{1}^{\prime}=1$.

(2) It follows directly from the definition of the order relation between two sequences.

(3) We have

$$
\begin{aligned}
l \underline{A}_{l} & =l 0 d_{1}^{\prime \prime} \ldots \leqslant \sigma^{j}\left(k \underline{A}_{k}\right) \\
& =l d_{0} d_{1} \ldots<\sigma^{j^{\prime}}\left(m \underline{A}_{m}\right)=l d_{0}^{\prime} d_{1}^{\prime} \ldots \leqslant l l d_{1}^{\prime \prime} \ldots
\end{aligned}
$$
obtain

Since $d_{0} \neq d_{0}^{\prime}$ it follows $d_{0}=0<1=d_{0}^{\prime}$. Then by (2) we

$$
\begin{aligned}
\sigma^{j^{\prime}+1}\left(m \underline{A}_{m}\right) & =\tau(l) d_{1}^{\prime} \ldots \leqslant \tau(l) d_{1}^{\prime \prime} \ldots \leqslant \sigma^{j+1}\left(k \underline{A}_{k}\right) \\
& =\tau(l) d_{1} \ldots
\end{aligned}
$$

as claimed.

Lemma 6.2. Let $\left[0 \underline{A}_{0}, \ldots,(n-1) \underline{A}_{n-1}\right]$ be an $n$-extremal block with cyclic permutation $\tau$. Let $k$ and $j$ be integers such that $k \in\{0, \ldots, n-1\}, j \geqslant 0$, $\tau^{j}(k)=l, \quad \sigma^{j}\left(k A_{k}\right)=l d_{0} d_{1} \ldots$ and $l A_{l}=l d_{0}^{\prime} d_{1}^{\prime} \ldots$. For each $m \geqslant 1$, set $r_{m}=\left|d_{0}^{\prime} \ldots d_{m-1}^{\prime}\right|_{1}$. Then we have:

(1) If $r_{m}=\left|d_{0} \ldots d_{m-1}\right|_{1}$ for some $m \geqslant 1$, then $\sigma^{\prime \prime \prime}\left(l A_{l}\right) \leqslant \sigma^{m+j}\left(k \underline{A}_{k}\right)$

(2) If $r_{m}+1=\left|d_{0} \ldots d_{m-1}\right|_{1}$ for some $m \geqslant 1$, then $\sigma^{m+j}\left(k A_{k}\right) \leqslant \sigma^{m}\left(l A_{l}\right)$.

(3) For all $m \geqslant 1$ we have $\left|d_{0} \ldots d_{m-1}\right|_{1} \in\left\{r_{m}, r_{m}+1\right\}$.

Proof. Since $\left[0 \underline{A}_{0}, \ldots,(n-1) \underline{A}_{n-1}\right]$ is an $n$-extremal block, $l A_{l}=l 0 d_{1}^{\prime} \ldots \leqslant \sigma^{j}\left(k A_{k}\right)=l d_{0} d_{1} \ldots \leqslant l 1 d_{1}^{\prime} \ldots$.

We start by proving (1). The equality $\left|d_{0} \ldots d_{m-1}\right|_{1}=r_{m}=\left|d_{0}^{\prime} \ldots d_{m-1}^{\prime}\right|_{1}$ tells us that for each $i \leqslant m-1$ such that $d_{i} \neq d_{i}^{\prime}$ there exists $j \leqslant m-1, j \neq i$ with the property that $d_{i}=d_{j}^{\prime}$ and $d_{i}^{\prime}=d_{j}$. Then, by Lemma 6.1(2) and (3), statement (1) holds. Statement (2) follows in a similar way.

Now we prove (3). If $l \underline{A}_{l}=\sigma^{j}\left(k \underline{A}_{k}\right)$, then $\left|d_{0} \ldots d_{m-1}\right|_{1}=\left|0 d_{1}^{\prime} \ldots d_{m-1}^{\prime}\right|_{1}$ for all $m \geqslant 1$, and we are done. Suppose $l \underline{A}_{l}<\sigma^{j}\left(k \underline{A}_{k}\right)$. Let $q$ be the smallest integer $\geqslant 0$ such that $\bar{d}_{q}^{\prime}=0 \neq 1=d_{q}$. Then $\left|d_{0} \ldots d_{j-1}\right|_{1}=\left|0 d_{1}^{\prime} \ldots d_{j-1}^{\prime}\right|_{1}$ for all $j \leqslant q$, and $\left|d_{0} \ldots d_{q}\right|_{1}=\left|1 d_{1}^{\prime} \ldots d_{q}^{\prime}\right|_{1}=r_{q}+1$. Now we apply $\sigma^{q+1}$ to the inequality $l A_{l}<\sigma^{j}\left(k A_{k}\right)$ and, from (2), we obtain $\sigma^{j+q+1}\left(k \underline{A}_{k}\right) \leqslant \sigma^{q+1}\left(l \underline{A}_{l}\right)$.

If $\sigma^{j+q+1}\left(k \underline{A}_{k}\right)=\sigma^{q+1}\left(l \underline{A}_{l}\right)$, then $\left|d_{0} \ldots d_{m-1}\right|_{1}$ $=\left|1 d_{1}^{\prime} \ldots d_{m-1}^{\prime}\right|_{1}$ for all $m \geqslant q+1$ and (3) follows. If $\sigma^{j+q+1}\left(k \underline{A}_{k}\right)<\sigma^{q+1}\left(l \underline{A}_{l}\right)$, let $p$ be the smallest integer larger than or equal to 1 such that $d_{q+p}=0 \neq 1=d_{q+p}^{\prime}$. Then $\left|d_{0} \ldots d_{j-1}\right|_{1}=\left|1 d_{1}^{\prime} \ldots d_{j-1}^{\prime}\right|_{1}$ for all $j \leqslant q+p$ and $\left|d_{0} \ldots d_{q+p}\right|_{1}=\left|0 d_{1}^{\prime} \ldots d_{q+p}^{\prime}\right|_{1}$. Now, in a similar way as above, from (1), it follows $\sigma^{q+p+1}\left(l \underline{A}_{l}\right) \leqslant \sigma^{q+p+1+j}\left(k \underline{A}_{k}\right)$.

In this way we can obtain (3) inductively.

Corollary 6.3. Let $k A_{k}=k d_{0} d_{1} \ldots$ be a sequence from an n-extremal block. Then $A_{k}$ is weightable of order $n$. Moreover, if for some non-negative integers $r$ and $s$ we have that $\sigma^{r n}\left(k \underline{A}_{k}\right) \leqslant \sigma^{s \prime}\left(k \underline{A}_{k}\right)$, then $\left|d_{r n} \ldots d_{(r+1) n-1}\right|_{1} \leqslant\left|d_{s n} \ldots d_{(s+1) n-1}\right|_{1}$. 
Proof. Let $r=\left|d_{1} \ldots d_{n-1}\right|_{1}$. We have to see that $\left|d_{i n} \ldots d_{(i+1) n-1}\right|_{1} \in\{r, r+1\}$ for all $i \geqslant 0$. We use Lemma 6.2(3) with $j=i n$ and $m=n$. Then we obtain $l=k$ because $\tau^{\prime \prime}=i d$ and, hence, $r_{n}=r$.

Since $\sigma^{i n}\left(k \underline{A}_{k}\right)=k d_{i n} d_{i n+1} \ldots$, then $\left|d_{i n} \ldots d_{(i+1) n-1}\right|_{1}$ $\in\{r, r+1\}$ and $A_{k}$ is weightable of order $n$.

Now suppose $\sigma^{r n}\left(k \underline{A}_{k}\right) \leqslant \sigma^{s n}\left(k A_{k}\right) \quad$ and $\left|d_{s n} \ldots d_{(s+1) n-1}\right|_{1}<\left|d_{r n} \ldots d_{(r+q) n-1}\right|_{1}$ and we shall obtain a contradiction. Indeed, then $\left|d_{s n} \ldots d_{(s+1) n-1}\right|=\omega_{n}\left(A_{k}\right)$, $\left|d_{r n} \ldots d_{(r+1) n-1}\right|_{1}=\omega_{n}\left(\underline{A}_{k}\right)+1$ and $\sigma^{r n}\left(k \underline{A}_{k}\right)<\sigma^{s n}\left(k \underline{A}_{k}\right)$.

Since $k A_{k}=k 0 d_{1} \ldots \leqslant \sigma^{r n}\left(k A_{k}\right)<\sigma^{s n}\left(k A_{k}\right) \leqslant k 1 d_{1} \ldots$, from $\omega_{n}\left(\underline{A}_{k}\right)=\left|0 d_{1} \ldots d_{n-1}\right|_{1}$ and from Lemma 6.1 it follows $\sigma^{n}\left(k A_{k}\right) \leqslant \sigma^{s n+n}\left(k A_{k}\right) \leqslant \sigma^{r n+n}\left(k \underline{A}_{k}\right) \leqslant \sigma^{n}\left(k \underline{A}_{k}\right)$. Therefore $\sigma^{(r+1) n}\left(k \underline{A}_{k}\right)=\sigma^{(s+1) n}\left(k \underline{A}_{k}\right)=\sigma^{n}\left(k \underline{A}_{k}\right)$. Then $\sigma^{n}\left(k \underline{A}_{k}\right)$ $=k\left(\underline{B}_{1} \ldots \underline{B}_{M}\right)^{\infty}$, where $\underline{B}_{i}=d_{i n} \ldots \bar{d}_{(i+1) n-1}$ for $1 \leqslant i \leqslant M$ and $M=\operatorname{gcd}(r, s)$. In this situation, the first $n$ elements of $\sigma^{r n}\left(k A_{k}\right)$ and of $\sigma^{s n}\left(k \underline{A}_{k}\right)$ must be equal to $\underline{B}_{M}$, in contradiction with our hypothesis.

The above corollary allows us to define a deflation operation $\Delta$ on the space of sequences from $n$-extremal blocks, in the following way:

$$
\Delta\left(k \underline{A}_{k}\right)=\Delta\left(d_{0} \ldots d_{n-1}\right) \Delta\left(d_{n} \ldots d_{2 n-1}\right) \ldots,
$$

where $\Delta\left(d_{r n} \ldots d_{(r+1) n-1}\right)=\left|d_{r n} \ldots d_{(r+1) n-1}\right|_{1}-\omega_{n}\left(\underline{A}_{k}\right)$ for every $r \geqslant 0$.

This deflation operation gives us a new admissible sequence $\Delta\left(k \underline{A}_{k}\right)$ for every $k$. We want to show that these sequences correspond to kneading invariants of rotations. First of all, as for the deflation operation of rotations, we shall prove that, in some sense, $\Delta$ commutes with $\sigma$.

Lemma 6.4. Let $k \underline{A}_{k}$ be a sequence from an n-extremal block and let $r$ and $s$ be non-negative integers such that $\sigma^{r \prime \prime}\left(k A_{k}\right) \leqslant \sigma^{s \prime \prime}\left(k \underline{A}_{k}\right)$. Then $\sigma^{r}\left(\Delta\left(k \underline{A}_{k}\right)\right) \leqslant \sigma^{s}\left(\Delta\left(k \underline{A}_{k}\right)\right)$.

Proof. Set $\sigma^{r n}\left(k \underline{A}_{k}\right)=k d_{r n} \ldots d_{r n+n-1} \ldots$ and $\sigma^{s n}\left(k \underline{A}_{k}\right)$ $=k d_{s n} \ldots d_{s n+n-1} \ldots$. Assume that $\left|d_{r n} \ldots d_{(r+1) n-1}\right|_{1}$ $\neq\left|d_{s n} \ldots d_{(s+1) n-1}\right|_{1}$. Then, from Corollary 6.3 , we get that

$$
\begin{aligned}
\left|d_{r n} \ldots d_{(r+1) n-1}\right|_{1} & =\omega_{n}\left(A_{k}\right)<\left|d_{s n} \ldots d_{(s+1) n-1}\right|_{1} \\
& =\omega_{n}\left(\underline{A}_{k}\right)+1 .
\end{aligned}
$$

Therefore $\sigma^{r}\left(\Delta\left(k \underline{A}_{k}\right)\right)=0 \ldots<1 \ldots=\sigma^{s}\left(\Delta\left(k \underline{A}_{k}\right)\right)$ as it was claimed.

If $\left|d_{r_{n}} \ldots d_{(r+1) n-1}\right|_{1}=\left|d_{s n} \ldots d_{(s+1) n-1}\right|_{1}$ then it follows that $\sigma^{r}\left(\Delta\left(k \underline{A}_{k}\right)\right)=d_{0}^{\prime} d_{1}^{\prime} \ldots$ and $\sigma^{s}\left(\Delta\left(k \underline{A}_{k}\right)\right)=d_{0}^{\prime} d_{1}^{\prime \prime} \ldots$, i.e., both sequences coincide at the first symbol. Furthermore, from Lemma 6.2(1), it follows that $\sigma^{(r+1) n}\left(k \underline{A}_{k}\right) \leqslant \sigma^{(s+1) n}\left(k \underline{A}_{k}\right)$ and we are in a situation which is similar to the initial one. Repeating the same argument in an iterative way, the lemma follows.

Proposition 6.5. Let $\left[0 \underline{A}_{0}, \ldots,(n-1) \underline{A}_{n-1}\right]$ be an n-extremal block. Then $\Delta\left(k \underline{A}_{k}\right)$ is extremal for each $k=0, \ldots, n-1$.

Proof. Since $k \underline{A}_{k}=k d_{0} d_{1} \ldots=k 0 d_{1} \ldots$, from Corollary 6.3 it follows that $\Delta\left(k \underline{A}_{k}\right)=0 \underline{A}$ for some admissible sequence $A$.

Now we shall prove that $\Delta\left(k \underline{A}_{k}\right)=d_{0}^{\prime} d_{1}^{\prime} \ldots$ satisfies condition (B), that is, $\Delta\left(k \underline{A}_{k}\right)=d_{0}^{\prime} d_{1}^{\prime} \ldots \leqslant \sigma^{r}\left(\Delta\left(k \underline{A}_{k}\right)\right)$ $=d_{r}^{\prime} d_{r+1}^{\prime} \ldots \leqslant 1 d_{1}^{\prime} \ldots$ for all $r \geqslant 0$. Since $k \underline{A}_{k} \leqslant \sigma^{r n}\left(k \underline{A}_{k}\right)$, from Lemma 6.4 , it follows that $\Delta\left(k \underline{A}_{k}\right) \leqslant \sigma^{r}\left(\Delta\left(k \underline{A}_{k}\right)\right)$. If $d_{r}^{\prime}=0$ then, obviously $\sigma^{\prime}\left(\Delta\left(k A_{k}\right)\right) \leqslant 1 d_{1}^{\prime} \ldots$. If $d_{r}^{\prime}=1$ then, by the definition of $\Delta\left(k A_{k}\right)$, we have $\left|d_{r n} \ldots d_{(r+1) n-1}\right|_{1}=\omega_{n}\left(\underline{A}_{k}\right)+1$. Now we claim that this equality is equivalent to the hypothesis of Lemma 6.2(2). Indeed, if in Lemma 6.2 we take $j=r n$ then, clearly, $k=l$. So, if in Lemma 6.2 we set $m=n$ we get that $r_{m}=\omega_{n}\left(A_{k}\right)$ and the claim is proved. Then, since $k A_{k} \leqslant \sigma^{\prime \prime \prime}\left(k A_{k}\right)$, by Lemma 6.2(2) we have $\sigma^{(r+1) n}\left(k \underline{A}_{k}\right) \leqslant \sigma^{\prime \prime}\left(k A_{k}\right)$. From Lemma 6.4 we obtain $\sigma^{(r+1)}\left(\Delta\left(k \underline{A}_{k}\right)\right) \leqslant \sigma\left(\Delta\left(k \underline{A}_{k}\right)\right)$. Therefore $\sigma^{r}\left(\Delta\left(k \underline{A}_{k}\right)\right)=1 \sigma^{r+1}\left(\Delta\left(k A_{k}\right)\right) \leqslant 1 \sigma\left(\Delta\left(k \underline{A}_{k}\right)\right)=1 d_{1}^{\prime} \ldots$.

We say that an $n$-extremal block is nonpreperiodic if $\Delta\left(k \underline{A}_{k}\right)$ is not preperiodic for each $k=0,1, \ldots, n-1$. Then, from the characterization of the kneading invariants of rotations (see Theorem 5.13) and from the above statements, we obtain as a coroliary the following theorem.

Theorem 6.6. Given a nonpreperiodic n-extremal block $\left[0 \underline{A}_{0}, \ldots,(n-1) \underline{A}_{n-1}\right]$, all the extremal sequences $\Delta\left(k A_{k}\right)$, for $k=0,1, \ldots, n-1$, are kneading invariants of rotations.

The next result shows that all the sequences $\Delta\left(k A_{k}\right)$, for $k=0,1, \ldots, n-1$ are in fact equal.

Theorem 6.7. $\left[0 A_{0}, \ldots,(n-1) A_{n-1}\right]$ be a nonpreperiodic n-extremal block. Then, for all $k=0,1, \ldots, n-1$ we have $\Delta\left(0 \underline{A}_{0}\right)=\Delta\left(k \underline{A}_{k}\right)$ and $\omega_{n}\left(\underline{A}_{0}\right)=\omega_{n}\left(A_{k}\right)$.

Proof. Thanks to Theorem 6.6, we know that all the extremal sequences $\Delta\left(k \underline{A}_{k}\right)$ for $k=0,1, \ldots, n-1$ are kneading invariants of rotations. It is well known that for a given kneading invariant of a rotation $0 d_{1} d_{2} \ldots$, the angle $\alpha$ of the rotation satisfies

$$
\alpha=\lim _{n \rightarrow \infty \infty} \frac{\left|0 d_{1} d_{2} \ldots d_{n-1}\right|_{1}}{n} .
$$

To see it we note that $d_{l}=E(l \alpha)-E((l-1) \alpha)$ for each $l \geqslant 1$. So,

$$
\frac{\left|0 d_{1} d_{2} \ldots d_{n-1}\right|_{1}}{n}=\frac{\sum_{i=1}^{n-1} d_{i}}{n}=\frac{E((n-1) \alpha)}{n} \rightarrow \alpha .
$$

Now, let $\gamma_{k}$ denote the angle of the rotation corresponding to the sequence $\Delta\left(k \underline{A}_{k}\right)$, where $A_{k}=0 d_{1}^{\prime} d_{2}^{\prime} \ldots$ and $\Delta\left(k A_{k}\right)=0 d_{1} d_{2} \ldots$. By Corollary 6.3 we know that the scquences $A_{k}$ are weightable of order $n$ with weight $\omega_{n}\left(\underline{A}_{k}\right)$. By cutting the sequence $A_{k}$ in blocks of length $n$, and by the definition of $\Delta$, we obtain

$$
\begin{gathered}
\left|0 d_{1}^{\prime} \ldots d_{n l-1}^{\prime}\right|_{1}=\sum_{j=0}^{l-1}\left|d_{j n}^{\prime} \ldots d_{j n+n-1}^{\prime}\right|_{1}=\sum_{j=0}^{l-1}\left(\omega_{n}\left(A_{k}\right)\right. \\
\left.+d_{j}\right)=l \omega_{n}\left(A_{k}\right)+\sum_{j=0}^{l-1} d_{j} .
\end{gathered}
$$

Consequently, $\quad \lim _{l \rightarrow \infty}(1 / l)\left|0 d_{1}^{\prime} \ldots d_{n l-1}^{\prime}\right|_{1}=\omega_{n}\left(A_{k}\right)+\gamma_{k}$. For any integer $j$, there exists an integer $p$ such that $p n \leqslant j$ $<(p+1) n$. Then we have 


$$
\begin{aligned}
\left|0 d_{1}^{\prime} \ldots d_{p n+n-1}^{\prime}\right|_{1}-j & \leqslant\left|d_{j}^{\prime} \ldots d_{j+n l-1}^{\prime}\right|_{1} \\
& \leqslant\left|0 d_{1}^{\prime} \ldots d_{(p+1) n+n-1}^{\prime}\right|
\end{aligned}
$$

and thus $\lim _{l \rightarrow \infty}(1 / l)\left|d_{j}^{\prime} \ldots d_{j+n l-1}^{\prime}\right|_{1}=\omega_{n}\left(\underline{A}_{k}\right)+\gamma_{k}$. On the other hand, since $\tau$ is a cyclic permutation we may assume that $\tau^{n-k}(k)=0$. Then, thanks to Lemma 6.2(3),

$\left|d_{n-k}^{\prime} \ldots d_{n-k+n l-1}^{\prime}\right|_{1} \in\left\{\left|d_{0}^{\prime \prime} \ldots d_{n-1-1}^{\prime \prime}\right|_{1},\left|d_{0}^{\prime \prime} \ldots d_{n l-1}^{\prime \prime}\right|_{1}+1\right\}$,

where $A_{0}=d_{0}^{\prime \prime} d_{1}^{\prime \prime} \ldots$. This yields

$$
\lim _{n \rightarrow \infty}(1 / l)\left|d_{n-k}^{\prime} \ldots d_{n-k}^{\prime}\right|_{1}=\omega_{n}\left(\underline{A}_{0}\right)+\gamma_{0}
$$

and, consequently, $\omega_{n}\left(\underline{A}_{k}\right)+\gamma_{k}=\omega_{n}\left(\underline{A}_{0}\right)+\gamma_{0}$ for all $k$. Since the $\omega_{n}\left(A_{k}\right)$ are integers and the $\gamma_{k}$ are numbers in $[0,1)$ we have $\omega_{n}\left(\underline{A}_{k}\right)=\omega_{n}\left(\underline{A}_{0}\right)$ and $\gamma_{k}=\gamma_{0}$ for all $k=0,1, \ldots, n-1$. This ends the proof of the theorem.

In the sequel we shall denote $\gamma_{0}=\gamma_{1}=\cdots=\gamma_{n-1}$ simply by $\gamma$ and $\omega_{n}\left(A_{0}\right)=\omega_{n}\left(A_{1}\right)=\cdots=\omega_{n}\left(\underline{A}_{k}\right)$ by $\omega$.

\section{KNEADING INVARIANTS}

From Sec. IV we already know that the kneading invariants of a composition of rotations in $n$ circles satisfy conditions $(A n)$ and $(B n)$. If we think in the case of the rotations, the nonpreperiodicity of an extremal block is the straightforward candidate to the property we need to complete the characterization of the kneading invariants of a composition of rotations. However, as the following example shows, a stronger condition is needed.

Example 7.1. Let.$/=\left(0 A_{0}, 1 A_{1}\right)$ be with $A_{0}=01(111110)^{\%}$ and $A_{1}=(011111)^{\%}$. It is not difficult to see that, if we take $\tau(i)=i+1(\bmod 2)$ for $i=0,1$, this is a 2-extremal block and that $\Delta\left(A_{0}\right)=\Delta\left(A_{1}\right)=(011)^{\%}$. Since $\Delta\left(\underline{A}_{0}\right)$ and $\Delta\left(\underline{A}_{1}\right)$ are not preperiodic then the block.$/$ is nonpreperiodic. Let us see that, indeed, . / is not the kneading invariant of any composition of rotations in 2-circles. Otherwise, there exists $f_{\tau, \sigma}$, having . $\%$ as the kneading invariant. Then, the address of $f_{\tau, \sigma}^{6}(0)$ is 1 . Hence, $f_{\tau, \sigma}^{6}(0)>0$. On the other hand, since $(011)^{x}$ is the kneading invariant of the rotation by angle $2 / 3$, the map $\left.f_{r, \sigma}^{2}\right|_{\{0.1)}$ is just this rotation. So, $\left(f_{\tau, \sigma}^{2}\right)^{3}(0)=f_{\tau, \sigma}^{\sigma}(0)=0$; a contradiction.

Next we state the stronger condition we need. Let $\left[0 \underline{A}_{0}, \ldots,(n-1) \underline{A}_{n-1}\right]$ be an $n$-extremal block. We say that it is strongly nonpreperiodic if each of the sequences $A_{k}$ for $k=0,1, \ldots, n-1$ is not preperiodic. Then we have the following theorem which is the main result of this paper.

Theorem 7.2. A kneading invariant of a composition of rotations in $n$ circles is an n-extremal block which is nonpreperiodic and strongly nonpreperiodic.

To prove this theorem we need to show first which is the geometrical meaning of the deflation operation on the kneading invariants of the composition of rotations in $n$ circles. We do this in the following proposition. We shall use the following notation. Let $\underline{I}(x)=k d_{0} d_{1} \ldots$ be the itinerary of a point by a composition of rotations in $n$ circles. Then the admissible sequence $d_{0} d_{1} \ldots$ will be denoted by $\hat{I}(x)$.

Proposition 7.3. Let $[\underline{I}(0), \ldots, \underline{I}(n-1)]$ be the kneading invariant of a composition of rotations in $n$ circles $f_{\tau, \alpha}$. Then the following holds: (a) $\omega=\omega_{n}(\hat{I}(0))=\cdots=\omega_{n}(\hat{I}(n-1))=E\left(\sum_{i=0}^{n-1} \bar{\alpha}(i)\right)$.

(b) For $k=0,1, \ldots, n-1$ the map $f_{\tau, \alpha}^{n} l_{(k, k+1)}$ is a rotation with angle $\gamma=\sum_{i=0}^{n-1} \bar{\alpha}(i)$ and kneading invariant $\Delta(\underline{I}(0))$ $=\Delta(\underline{I}(1))=\cdots=\Delta(\underline{I}(n-1))$.

Proof. First we prove (b). For $l \geqslant 0$ we have

$$
f_{\tau, \alpha}^{\prime}=f_{\tau^{l}, \alpha+\alpha^{\circ} \tau+\cdots+\psi^{\circ} \tau^{i-1}} \quad(*) .
$$

Since $\tau$ is a cyclic permutation, $\tau^{\prime \prime}=i d$ and hence $\left.f_{\tau, \alpha}^{n}\right|_{\mid k, k+1)}$ is a rotation of angle $\left(\alpha+\alpha^{\circ} \tau+\cdots+\alpha^{\circ} \tau^{\prime-1}\right)(k)$ for all $k=0, \ldots, n-1$. Now we prove that the kneading invariant of such rotation is $\Delta(I(0))$.

Denote by $d_{0}^{\prime} d_{1}^{\prime} \ldots$ the kneading invariant of $f_{\tau, \alpha}^{n} l_{(k, k+1)}$. Then, in view of the definition of address, $d_{m}^{\prime}$ is 0 if $f_{\tau_{,}(x)}^{\prime \prime \prime}(k)$ is smaller than $k+1-\alpha$ and 1 otherwise, where $\alpha$ $=\sum_{i=0}^{n-1} \bar{\alpha}\left(\tau^{i}(k)\right)-E\left[\Sigma_{i=0}^{n-[} \bar{\alpha}\left(\tau^{i}(k)\right)\right]$.

By $(*)$, we have

$$
f_{\tau, \alpha}^{m n}(k)=\tau^{m n}(k)+\sum_{i=0}^{m n-1} \bar{\alpha}\left(\tau^{i}(k)\right)-E\left(\sum_{i=0}^{m n-1} \bar{\alpha}\left(\tau^{i}(k)\right)\right)
$$

Therefore,

$$
f_{\tau, \alpha x}^{m n}(k)=k+m \sum_{i=0}^{n-1} \bar{\alpha}\left(\tau^{i}(k)\right)-E\left(m \sum_{i=0}^{n-1} \bar{\alpha}\left(\tau^{i}(k)\right)\right),
$$

because $\tau^{n}=i d$.

For shortness denote $\sum_{i=0}^{n-1} \bar{\alpha}\left(\tau^{i}(k)\right)$ by $\beta$. Hence $d_{m}^{\prime}$ is 0 if $(m+1) \beta-E(m \beta)<1+E(\beta)$ and 1 otherwise. Set $\Delta(I(k))=d_{0}^{\prime \prime} d_{1}^{\prime \prime} \ldots$, then

$$
\begin{aligned}
d_{m}^{\prime \prime}= & \Delta\left(d_{m n} \ldots d_{(m+1) n-1}\right)=\left|d_{m n} \ldots d_{(m+1) n-1}\right|_{1} \\
& -\omega_{n}\left(\underline{A}_{k}\right)=\left|d_{m n} \ldots d_{(m+1) n-1}\right|_{1}-\left|d_{0} d_{1} \ldots d_{n-1}\right|_{1} .
\end{aligned}
$$

Hence, by Proposition 4.2 and the fact that $\left|d_{m n} \ldots d_{(m+1) n-1}\right|_{1}=\sum_{i=0}^{n-1} d_{m n+i}$, we obtain

$$
d_{m}^{\prime \prime}=E((m+1) \beta)-E(m \beta)-E(\beta) .
$$

Therefore, $d_{m}^{\prime \prime}$ is 0 if $E((m+1) \beta)-E(m \beta)$ is $E(\beta)$ and 1 if $E((m+1) \beta)-E(m \beta)$ is $E(\beta)+1$. Since

$$
\begin{aligned}
E((m+1) \beta)-E(m \beta) & \leqslant(m+1) \beta-E(m \beta) \\
& <E((m+1) \beta)-E(m \beta)+1,
\end{aligned}
$$

then $d_{m}^{\prime}$ and $d_{m}^{\prime \prime}$ must coincide. Thus, the kneading invariant of $\left.f_{\tau, \alpha}^{\prime \prime}\right|_{[k, k+1)}$ is $\Delta(I(k))$ for each $k=0,1, \ldots, n-1$. Since $\left.f_{\tau, \alpha}^{n}\right|_{(k, k+1)}$ are the same rotation for all $k$, it follows that their kneading invariants are all $\Delta(I(0))$.

Now we prove (a). In view of (b) and Theorem 5.13, $[\underline{I}(0), \ldots, \underline{I}(n-1)]$ is nonpreperiodic. Then, thanks to Theorem 6.7 it is enough to compute $\omega(\hat{I}(0))$. We proceed by induction. Set $\hat{I}(0)=0 d_{1} d_{2} \ldots$. Then $|0|_{1}=0=E(\bar{\alpha}(0))$. Assume now that $\left.\overline{\mid 0} d_{1} \ldots d_{l}\right|_{1}=E\left[\Sigma_{i=0}^{l} \bar{\alpha}(i)\right]$ for some $\bar{l} \geqslant 0$. We shall prove that $\left|0 d_{1} \ldots d_{l+1}\right|_{1}=E\left[\sum_{i=0}^{l+1} \bar{\alpha}(i)\right]$. This will end the proof of the lemma. As in the proof of Proposition 4.2 we 
have that $d_{l+1}=1$ if and only if $\sum_{i=0}^{l} \bar{\alpha}(i)$ $-E\left[\sum_{i=1}^{l} \bar{\alpha}(i)\right] \geqslant 1-\bar{\alpha}(l+1)$. That is, $d_{l+1}=1$ if and only if $\sum_{i=0}^{l+1} \bar{\alpha}(i) \geqslant E\left[\sum_{i=0}^{l} \bar{\alpha}(i)\right]+1$. Consequently, if $d_{l+1}=1$ we have $E\left[\sum_{i=0}^{l+1} \bar{\alpha}(i)\right]=E\left[\Sigma_{i=0}^{l} \bar{\alpha}(i)\right]+1$ and then $\left|0 d_{1} \ldots, d_{l+1}\right|_{1}=E\left[\Sigma_{i=0}^{l+1} \bar{\alpha}(i)\right]$. If $d_{l+1}=0$, then $E\left[\sum_{i=0}^{i+i} \bar{\alpha}(i)\right]=E\left[\sum_{i=0}^{i} \bar{\alpha}(i)\right]$ and hence $\left|0 d_{1} \ldots d_{l+1}\right|$ i $=E\left[\sum_{i=0}^{Y+1} \bar{\alpha}(i)\right]$.

Proof of Theorem 7.2. The extremality and the nonpreperiodicity follow from Propositions 4.3 and 7.3, and Theorem 5.13. Let us prove the strongly nonpreperiodicity. Assume that $[I(0), \ldots, \underline{I}(n-1)]$ is the kneading invariant of a composition of rotations in $n$ circles $f_{\tau, \alpha}$. We suppose that it is not strongly nonpreperiodic and we shall obtain a contradiction.

Suppose that there is some $k \in\{0,1, \ldots, n-1\}$ such that $\underline{I}(k)=k d_{0} \ldots d_{m}\left(d_{m+1} \ldots d_{m+p}\right)^{\infty}$ with $d_{m} \neq d_{m+p}$ for some $m \geqslant 0$ and $p \geqslant 1$. Let $l=\tau^{\prime \prime}(k)$. From the extremality of the kneading invariant it follows that

$$
\underline{I}(l)=l \underline{I}(l) \leqslant \sigma^{i}(\underline{I}(k))=l d_{i}\left(d_{m+1} \ldots d_{m+p}\right)^{\infty} \leqslant l 1 \sigma(\hat{I}(l))
$$

for $i$ equals to $m$ and $m+n p$. Since $d_{m} \neq d_{m+p}=d_{m+n p}$, either $d_{m}$ or $d_{m+n p}$ is 0 and the other symbol is 1 . Thus, by applying $\sigma$ to the above inequalities, from Lemma 6.1 we obtain that $\left.\left(d_{m+1} \ldots d_{m+p}\right)\right)^{\infty} \leqslant v(\hat{I}(l)) \leqslant\left(d_{m+1} \ldots d_{m+p}\right)^{x}$. Then $\underline{I}(l)=l 0\left(d_{m+1} \ldots d_{m+p}\right){ }^{x}$ and so, $\Delta(\underline{I}(l))$ is eventually periodic. On the other hand, from Proposition 7.3 it follows that $\Delta(I(l))$ is the kneading invariant of the rotation $\left.f_{\tau, \alpha}^{\prime \prime}\right|_{\mid l, l+1)}$. Then, from Theorem $5.13, \Delta(\underline{I}(l))$ is a periodic sequence $\left(0 b_{1} \ldots b_{q-1}\right)^{x}$ for some $q \geqslant 1$ and $\left.f_{\tau, \alpha}^{n}\right|_{\mid, l+1)}$ is a rotation by a rational angle. Therefore, in view of Ref. 1, all the points in $[l, l+1)$ have one of the sequences $\sigma^{j}\left(\left(0 b_{1} \ldots b_{q-1}\right)^{\infty}\right)$ for $j=0,1, \ldots, q-1$ as itinerary under $f_{\left.\tau_{i}(v) ! l_{i} !+!\right)}^{n}$

From the fact that either $d_{m}=0$ or $d_{m+n p}=1$ or conversely and since $\underline{I}\left(f_{\tau, \alpha}^{i}(k)\right)=\sigma^{i}(\underline{I}(k))$ $=l d_{i}\left(d_{m+1} \ldots d_{m+p}\right)^{x}$ with $i \in\{m, m+n p\}$, there exists a point $x$ in $[l, l+1)$ having itinerary $l 1\left(d_{m+1} \ldots d_{m+p}\right)^{\infty}$ under $f_{\tau, \alpha}$. Thus, from the definition of $\Delta$ it follows that the $f_{\tau, \alpha^{-}}^{n}$ itinerary of $x$ is $\left(1 b_{1} \ldots b_{q-1}\right)^{x}$. But, since $\left|1 b_{1} \ldots b_{q-1}\right|_{1} \neq\left|0 b_{1} \ldots b_{q-1}\right|_{1}$, we get that $\left(1 b_{1} \ldots b_{q-1}\right)^{x} \neq \sigma^{j}\left(\left(0 b_{1} \ldots b_{q-1}\right)^{\infty}\right)$ for all $j=0,1, \ldots, q$ -1 ; a contradiction.

\section{ON THE CONVERSE OF THEOREM 7.2}

In the previous sections we have shown that each kneading invariant of a composition of rotations in $n$ circles is an $n$-extremal block which is strongly nonpreperiodic and nonpreperiodic. To show that these conditions characterize indeed the kneading invariantis of the compositions of rotations in $n$ circles we have to show the converse. To do this we propose the following procedure.

We shall start by defining a deflation operation $\rho$ on the space of sequences of $n$-extremal blocks which can be though of as a "local" version of $\Delta$.

Let . $\ell=\left[0 \underline{A}_{0}, \ldots,(n-1) A_{n-1}\right]$ be an $n$-extremal block with a cyclic permutation $\tau$. Set $k A_{k}=k d_{0}^{k} d_{1}^{k} \ldots$ for all $k=0,1, \ldots, n-1$ and $\pi=\left|d_{0}^{\tau^{n-2}(0)}, d_{1}^{\tau^{\prime-2}(0)}\right|_{1}=d_{1}^{\tau^{n-2}(0)}$. Then, for $k=\tau^{j}(0)$ with $j=0,1, \ldots, n-2$, we define

$$
\begin{aligned}
\rho\left(k \underline{A}_{k}\right)= & \rho(k) d_{0}^{k} d_{1}^{k} \ldots d_{n-j-3}^{k} \\
& \rho\left(d_{n-j-2}^{k}, d_{n-j-1}^{k}\right) d_{n-j}^{k} \ldots d_{2 n-j-3}^{k} \\
& \rho\left(d_{2 n-j-2}^{k}, d_{2 n-j-1}^{k}\right) d_{2 n-j}^{k} \ldots
\end{aligned}
$$

where $\rho\left(d_{m=j=2}^{k}, d_{m=j=1}^{k}\right)=\left|d_{m=j-2}^{k}, d_{i n-j-1}^{k}\right|_{1}-\pi$ for all $r \geqslant 1$ and

$$
\rho(k)=\left\{\begin{array}{l}
k, \quad \text { if } \quad k<\tau^{n-1}(0), \\
k-1, \quad \text { if } k>\tau^{n-1}(0) .
\end{array}\right.
$$

Let $l=\tau^{n-1}(0)$. From Lemma $6.2(3)$ it follows that $\rho\left(k \underline{A}_{k}\right) \in\{\rho(k)\} \times\{0,1\}^{\mathrm{N}}$ for each $k \neq l$. We denote $\left[\rho\left(0 \underline{A}_{0}\right), \ldots, \rho\left((l-1) \underline{A}_{l-1}\right), \rho\left((l+1) \underline{A}_{l+1}\right), \ldots, \rho\left((n-1) \underline{A}_{n-1}\right)\right]$ by $\rho($. $)$. Then the following can be proved straightforwardly

Proposition 8.1. Let,$l=\left[0 A_{0}, \ldots,(n-1) A_{n-1}\right]$ be an n-extremal block with a cyclic permutation $\tau$. Then

(1) $\rho(.$,$) is an (n-1)$-extremal block with cyclic permutation $\tau^{\prime}$ such that $\tau^{\prime}(\rho(k))=\rho(\tau(k))$ for all $k \neq \tau^{n-2}(0)$ and $\tau^{\prime}\left[\rho\left(\tau^{n-2}(0)\right)\right]=0$.

(2) $\rho^{n-1}\left(. l_{1}\right)=\Delta\left(k A_{k}\right)$ for all $k=0,1, \ldots, n-1$.

Moreover, we conjecture the following

Conjecture 8.2. If . $\%$ is an n-extremal strongly nonpreperiodic block, then $\rho(.$,$) is also strongly non-$ preperiodic.

We note that in the case of rotations the notion of strongly nonpreperiodicity coincides with the notion of nonpreperiodicity. Therefore, as an immediate consequence of the above conjecture and proposition we obtain

Corollary 8.3. Any n-extremal strongly nonpreperiodic block is nonpreperiodic.

Moreover, Conjecture 8.2 shows us a possible strategy to prove the converse of Theorem 7.2. This strategy is based in the following new conjecture.

Conjecture 8.4. Let . 1 , be an n-extremal nonpreperiodic block. If $\rho(.$,$) is the kneading invariant of a composition of$ rotations, then so is. . .

Finally, if the above two conjectures hold, we obtain the following characterization

Theorem 8.5. A block. \% is the kneading invariant of a composition of rotations in $n$ circles if and only if it is n-extremal and strongly nonpreperiodic.

Concluding Remark. As it has been said in Secs. I and III the coding at the level of the composition of rotations in $n$ circles is easily translated to the natural coding of curves in a tubuilar neighborhood $U$ of the heterochinic cycle. Thus, Theorem 7.2 and the results and conjectures in this section characterize the coding of the unstable branches of the singularities $p_{0}, p_{1}, \ldots, p_{n-1}$ for the perturbed vector field satisfying $\mathrm{H}_{1}$ to $\mathrm{H}_{6}$ in $U$. The big open question is to describe these codings in the general case, that is, if we do not restrict the allowed perturbations to the $n$-dimensional subvariety of the parameter space described by $\mathrm{H}_{2}$ through $\mathrm{H}_{6}$. 


\section{ACKNOWLEDGMENTS}

Jean Marc Gambaudo kindly thanks the Centre de Recerca Matemàtica for their support while this paper was being written. Lluís Alsedà has been partially supported by the DGICYT Grant No. PB90-0695. We thank the referees of the paper for careful reading and many suggestions which improved the writing of the paper.
'J. M. Gambaudo, "Ordre, désordre, et frontière des systèmes MorseSmale," Thèse d'ctat, Universite de Nicc, 1987.

${ }^{2}$ J. M. Gambaudo, P. Glendinning, and C. Tresser, Nonlinearity 1, 203 (1988).

${ }^{3}$ J. M. Gambaudo and C. Tresser, Bol. Soc. Bras. Mat. 19, 61 (1988).

${ }^{4}$ S. A. Campbell and P. Holmes, Nonlinearity 4, 697 (1991).

${ }^{5}$ M. Morse and G. A. Hedlund, Am. J. Math. 62, I (1940). 